\title{
In-situ thermography for laser powder bed fusion: effects of layer temperature on porosity, microstructure and mechanical properties
}

\author{
Richard J. Williams ${ }^{\mathrm{a}}$, Alessandro Piglione ${ }^{\mathrm{b}}$, Tobias Rønneberg ${ }^{\mathrm{a}}$, Connor Jones ${ }^{\mathrm{c}}$, \\ Minh-Son Pham ${ }^{\mathrm{b}}$, Catrin M. Davies ${ }^{\mathrm{a}}$, Paul A. Hoopera,* \\ ${ }^{a}$ Department of Mechanical Engineering, Imperial College London. SW7 2AZ, UK. \\ ${ }^{b}$ Department of Materials, Imperial College London. SW7 2AZ, UK. \\ ${ }^{c} A W E$ plc, Aldermaston, Reading. RG7 $4 P R, U K$.
}

\begin{abstract}
In laser powder bed fusion (LPBF) the surface layer temperature is continually changing throughout the build process. Variations in part geometry, scanned cross-section and number of parts all influence the thermal field within a build. Process parameters do not take these variations into account and this can result in increased porosity and differences in local microstructure and mechanical properties, undermining confidence in the structural integrity of a part. In this paper a wide-field in-situ infra-red imaging system is developed and calibrated to enable measurement of both solid and powder surface temperatures across the full powder bed. The influence of inter-layer cooling time is investigated using a build scenario with cylindrical components of differing heights. In-situ surface temperature data are acquired throughout the build process and are compared to results from porosity, microstructure and mechanical property investigations. Changes in surface temperature of up to $200^{\circ} \mathrm{C}$ are attributed to variation in inter-layer cooling time and this is found to correlate with density and grain structure changes in the part. This work shows that these changes are significant and must be accounted for to improve the consistency and structural integrity of LPBF components.
\end{abstract}

Keywords: in-situ monitoring, infra-red, powder bed fusion, porosity, temperature

\section{Introduction}

Laser powder bed fusion (LPBF) is currently the most widely adopted additive manufacturing (AM) method for producing metal components. While this uptake has been driven by the many potential benefits of AM processes, it has also been held back by incomplete process understanding and a lack of confidence in the structural integrity of components produced $[1,2]$. This is understandable given the complexity of the process and the many input process parameters that need to be correctly set and controlled to

\footnotetext{
* Corresponding author

Email address: paul.hooper@imperial.ac.uk (Paul A. Hooper)
}

Preprint submitted to Additive Manufacturing 
achieve a satisfactory end result. Even with the perfect set of parameters defects are unavoidable and may appear in unexpected places. In-situ process monitoring tools can help us to establish why this happens and how it can be mitigated $[1,3,4]$.

Within the build process the thermal fields in a component are continually changing. Local to the melt pool the temperature changes are rapid, transient and extreme. Further from the melt pool, at the part-scale or continuum-scale, the temperature field changes are more gradual and less extreme in nature. The local temperature field is closely linked to the laser input parameters (power, speed, scan path, etc) whereas the part-scale temperature field is more closely linked to the part geometry, including any support structures which affect the heat flow paths [5, 6]. This part-scale temperature field, particularly at the build surface, is significant as it represents the starting temperature before the laser passes over the surface. Variations in this starting surface temperature are not accounted for as process parameter sets are effectively constant throughout a build. Too high a starting temperature will drive the melt pool towards key-hole mode [7]. Too low a starting temperature will decrease the melt pool size, leading to lack of fusion porosity $[8,9]$. In this paper the first calibrated full-field in-situ surface layer temperature measurements in LPBF are reported to investigate these effects, along with its effect on porosity, microstructure and mechanical properties.

The LPBF process excels at manufacturing complex geometries. When sliced into 2dimensional layers, the layers often possess greatly varying cross-sectional areas throughout the build, meaning each layer takes a different length of time to process. Furthermore parts are often nested together in a single build in order to improve machine utilisation, including parts of differing heights. The length of time between successive laser scans of a point on the cross-section varies layer-to-layer, effecting the cooling time of the surface layer. This inter-layer cooling time (ILCT) influences the average rate of heat input into the part, changing the thermal field. It also means multiple parts on the same build can influence each other's thermal histories. Since the process is fundamentally thermally driven, differing thermal histories in parts will also influence the mechanical properties and microstructure $[2,10,11,12]$.

This phenomenon has not been widely investigated in literature for LPBF. Some effects of ILCT have been reported in directed energy deposition (DED) [13, 14, 15]. In particular, Yadollahi et al. found some correlation between the ILCT and porosity, Vicker's hardness and compressive strength in DED. However, the same authors proposed in [16] that ILCT was a less important factor in LPBF due to the differing thermal and temporal conditions between the two processes, in particular the far shorter inter-layer times associated with LPBF. Lui et al. [17] varied the ILCT in LPBF when processing Ti-6Al-4V cylinders with an irregular layer thickness of $90 \mu \mathrm{m}$. By manipulating the ILCT between 1-12s they were able to achieve favourable lamellar microstructure and found that the $\alpha$-lath width and morphology were sensitive to variation in ILCT. In a prior publication the same authors found minor differences in yield strength and ductility according to ILCT [18] and attributed this to a variation in lath width, following a HallPetch type relationship. The different lath widths would point to different cooling rates in the samples under different thermal conditions but no temperature measurements were conducted. Ti-6Al- $4 \mathrm{~V}$ is also a significantly different material system to other common LPBF materials (such as 316L) and contains distinct features in the AM microstructure. In all of the works identified here the ILCT is changed between builds, such that the component is built with a constant ILCT throughout the process. In real applications, 
where components have complex geometries and are nested in a single build, achieving a constant ILCT during the build is not possible. A change in ILCT during a build process is an important consideration and has not yet been examined in the LPBF literature.

While some of the papers on the ILCT variation in DED include quantification of the effects on part temperature, none exist for LPBF as it is a much more challenging task. Non-contact monitoring methods, such as infra-red (IR) thermography, provide the most straightforward opportunity to achieve this. The area of in-situ monitoring in LBPF has seen a great deal of attention recently and there are now examples in the literature of taking part-scale thermal measurements of the process using IR thermography $[19,20,21]$. However these frequently lack calibration and do not report absolute temperature. They are primarily used for process control, using arbitrary intensity values measured via IR as a control variable. Boone et al. [20] were able to achieve calibrated temperature measurements in Electron Beam Melting (EBM), although information on the uncertainty is limited.

In this paper, the effects of varying ILCT in LPBF are studied through test build scenarios involving the manufacture of cylinders in 316L stainless steel. An IR thermal imaging setup is integrated into a Renishaw AM250 LBPF machine and calibrated to measure surface temperature of solid and powder, along with estimates of uncertainty. In-process surface temperature measurements are presented along with optical microscopic images and EBSD to examine the corresponding influence on porosity and microstructure. Understanding how the process reacts to such inputs is essential to work towards achieving a repeatable process and building confidence in the structural integrity of LBPF components.

The paper is organised as follows. First a new in-situ full-field IR thermography system is described along with the procedures performed to calibrate temperature values and estimate uncertainty. This is followed by a definition of ILCT and the build scenarios used to investigate its effect on porosity, microstructure and mechanical properties. The results and discussion section then reports the surface temperature measurements made and the influence of ILCT on them. Differences in melt pool behaviour are then described using a previously developed high-speed melt pool imaging system [5]. Porosity, microstructure and mechanical properties are then reported and links to surface temperature and ILCT are established. Implications for process parameter development and component qualifification are briefly discussed before conclusions are drawn.

\section{Methods}

\subsection{In-situ full-field IR monitoring}

An in-situ thermal imaging setup is used to make full-field surface temperature measurements of the whole build area throughout the build process. A FLIR A35 IR camera is integrated within the build chamber of a Renishaw AM250 LPBF machine. This can be seen in Figure 1. The camera is located within a vacuum tight aluminium box to separate it from the processing area and views the build area through a germanium window. The camera points downwards, viewing the build plate at an angle of $66^{\circ}$ elevation from the horizontal and allows the whole build area to be imaged. Images are captured at a resolution of $320 \times 256$ pixels, equating to a pixel size of approximately $1 \mathrm{~mm}^{2}$, and recorded at a rate of $60 \mathrm{~Hz}$ for the entire build duration. Due to the spatial and 
temporal resolution of the camera, the melt pool itself cannot be viewed and instead the temperatures captured are at a lower range and occurring across longer time and length scales.

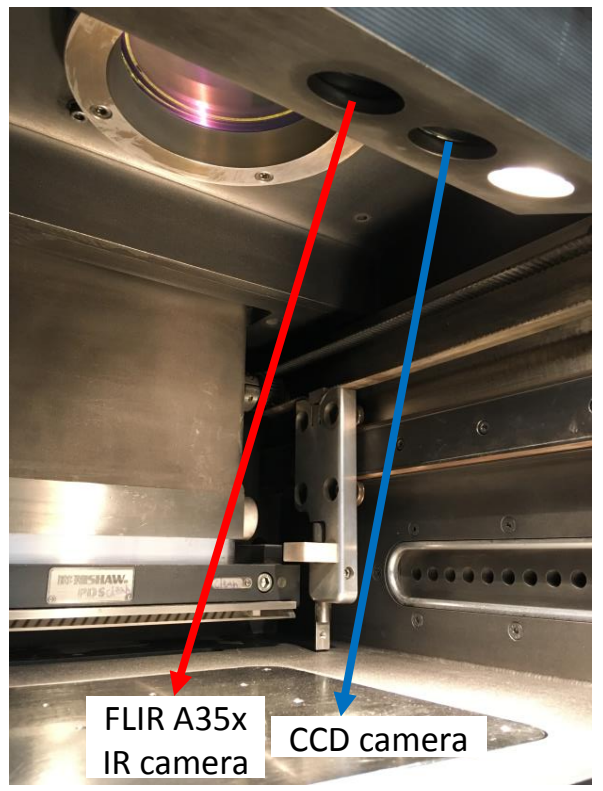

(a)

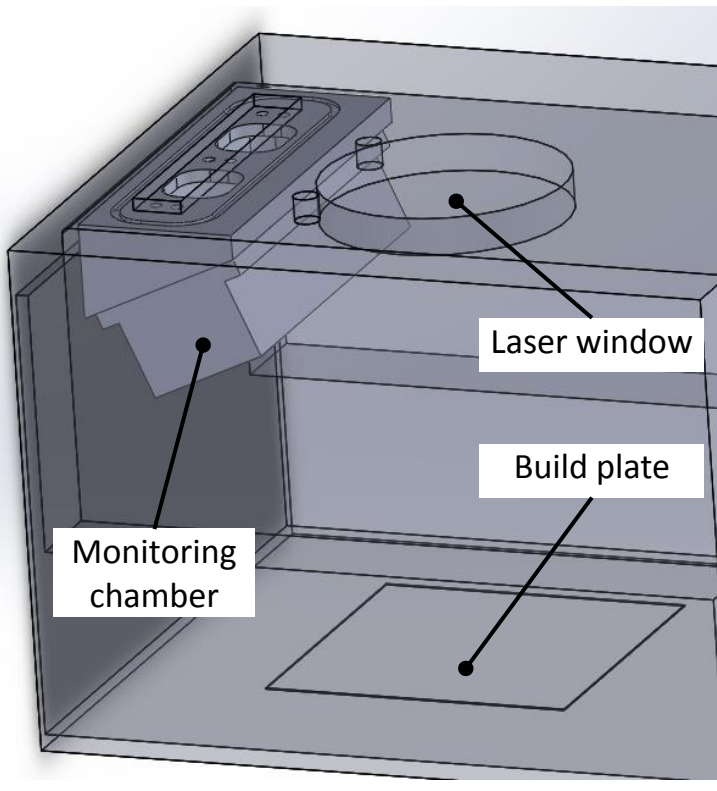

(b)

Figure 1: (a) A photograph of the modifications made to the Renishaw AM250; (b) A CAD model of the modifications, with the existing build chamber rendered as transparent

\subsubsection{Calibration}

Thermal imaging cameras are typically calibrated using a set of near-ideal blackbody sources at known temperatures. Although this calibrates the camera sensor's non-linear response and absolute magnitude well, the largest uncertainty in most applications is the emissivity of the target object. Emissivity is the efficiency of a body at emitting thermal radiation compared to a blackbody and must have a value between 0 and 1 . The emissivity of any real object is dependent on material, wavelength, temperature, surface roughness, oxidation of surface and view angle, among other things. Accurate emissivity values are difficult to measure, often being specific to a particular measurement scenario and introduce errors in the calculated temperatures. Additional optical elements in the optical path (a Ge window in this case) also render the manufacturer's calibration invalid. To avoid these complications a simpler empirical approach was used to calculate temperature from the IR camera's response.

A calibration component was designed and manufactured using the same machine, material and parameters as the components to be investigated. This ensured that the calibration component had a surface finish, and hence emissivity, representative of real components being built. The calibration component contained a recess to house a $200 \mathrm{~W}$ cartridge heater (manufactured by Acim Jouanin) and K-type thermocouples were at- 
tached to the top surface of the calibration component to enable control of surface temperature. The calibration component was located in the build chamber at the same position where surface temperatures were to be measured in the real component and was heated up. During heating the IR surface emissions were recorded on the camera and data was logged from the attached calibrated thermocouple. Recording also continued during cooling to check for any hysteresis in camera intensity due to oxidation (the calibration was performed under argon atmosphere).This calibration approach allows an empirical relationship to be derived between surface temperature and the intensity recorded by the IR camera. Any changes to emissivity due to temperature are contained within the empirical calibration curve. The drawback of this method is that it is specific to this experimental setup and changes to material, optical path, position, etc. require the calibration experiment to be repeated.

The uneven surface finish characteristic of LPBF parts can cause significant pixel-topixel variation in the intensity values observed as different pixels have different apparent emissivity. To mitigate this, a $9 \times 9$ box of pixel values on the component surface were extracted from the camera data, enabling a mean value to be calculated along with the standard deviation in the box. This gives an indication of the amount of variation present in the extracted box and allows the reliability of the mean value to be assessed. A calibration function is then evaluated, mapping the mean raw pixel intensity to an absolute temperature. The experiment is then repeated with unmelted powder on top of the calibration object to gather the same data with the emissivity difference of the powder particles. The component and experimental setup can be seen in Figure 2, along with an example IR intensity image from the calibration experiment.

The calibration curve for the solid surface is derived by plotting temperature recorded by the thermocouple against the mean camera pixel intensity and is shown in Figure 3(a). The error in the temperature measurement associated with the pixel-to-pixel variability is then quantified by computing the standard deviation and $2 \sigma$ (or 95\%) confidence interval as defined by standard error in the mean methodology . These parameters are plotted against temperature in Figure 3(b). The analysis was then repeated for the powder surface data to derive the powder calibration curve shown in Figure 4. From the two figures it is apparent that the pixel-to-pixel variation is much greater for the solid surface than for the powder surface. This shows that emissivity variation on the solid surface is detectable by the camera at this spatial resolution, whereas the powder surface behaves more uniformly since the powder particles are much smaller than the pixel size. Despite the standard deviation of the solid calibration being greater, both are still acceptable given the large number of pixels sampled. As expected, the higher surface roughness and hence emissivity of the powder layer produced a much flatter calibration function, mapping a given raw pixel intensity to a lower temperature value. 


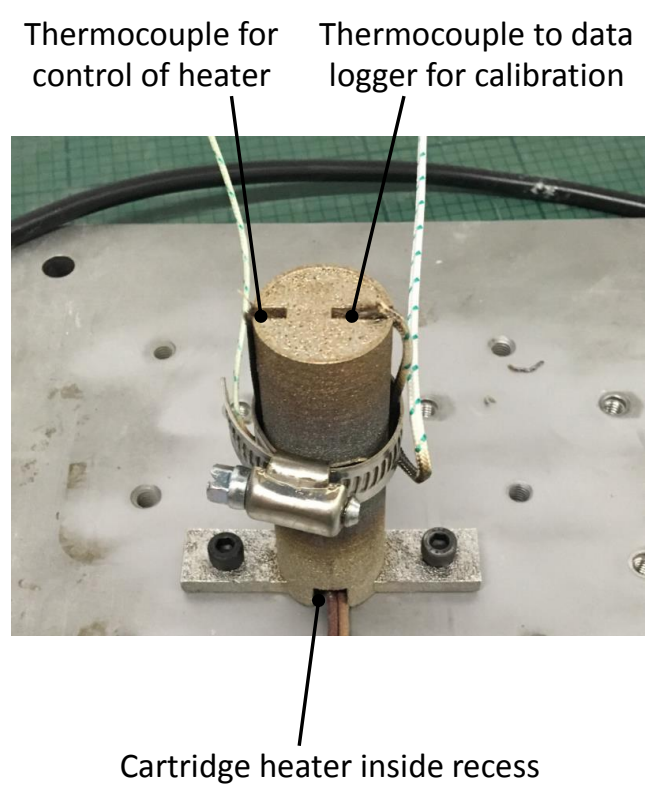

(a)

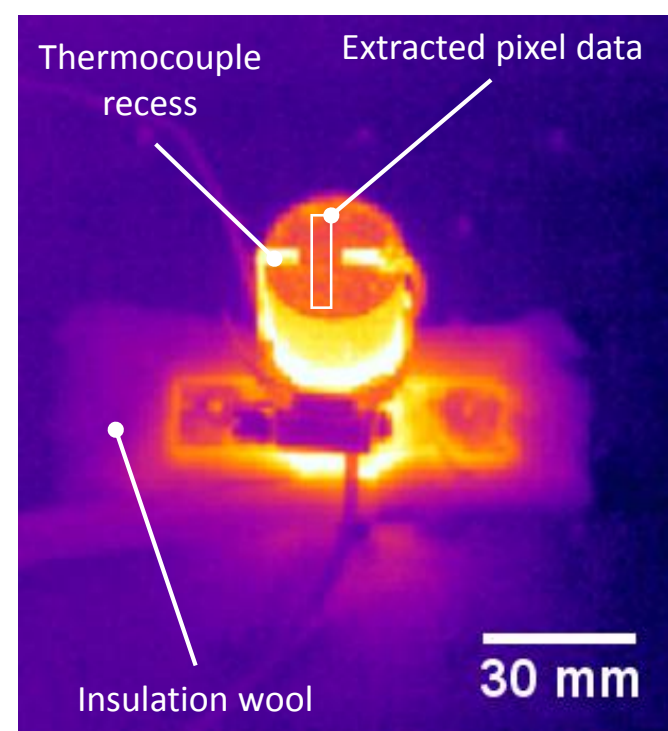

(b)

Figure 2: (a) A photograph of the calibration experimental setup; (b) An example emission intensity image taken from the IR camera during the calibration experiments

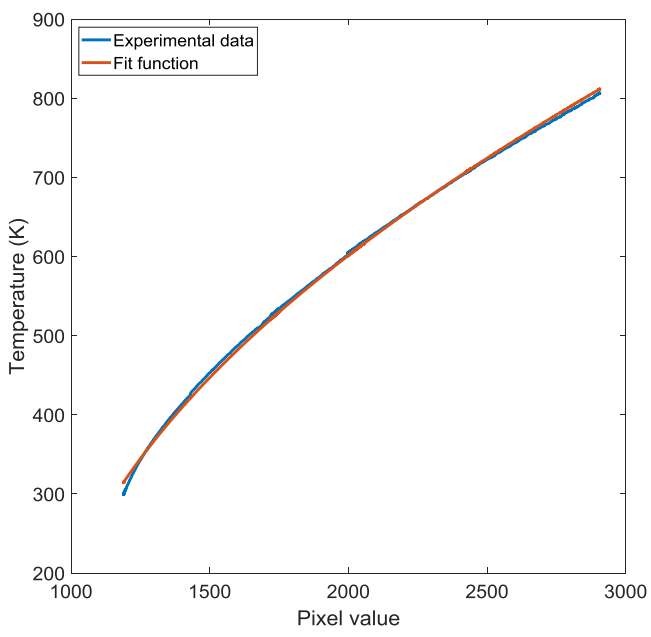

(a)

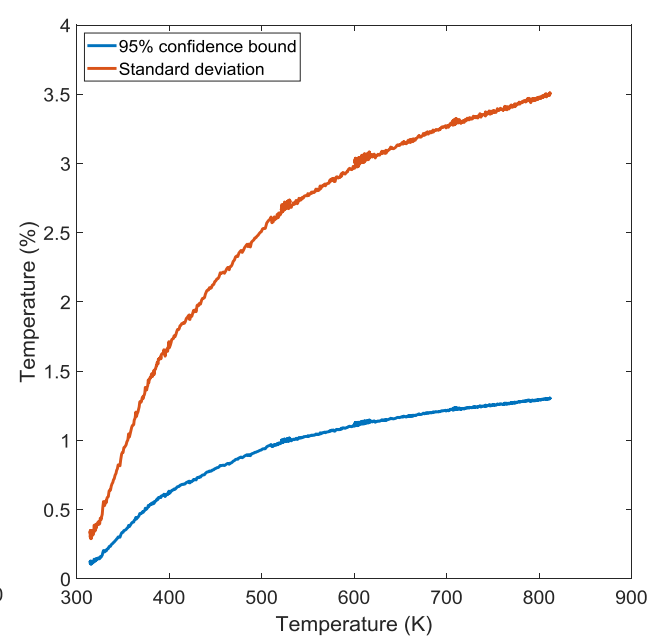

(b)

Figure 3: (a) A plot of the calibration curve for the solid surface emissivity with fit to the experimental data; (b) Quantification of the uncertainty in the temperature measurement vs temperature 


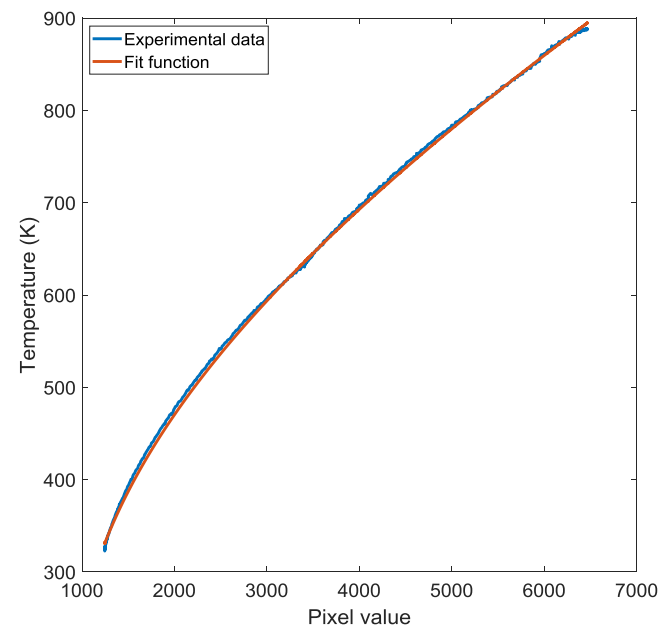

(a)

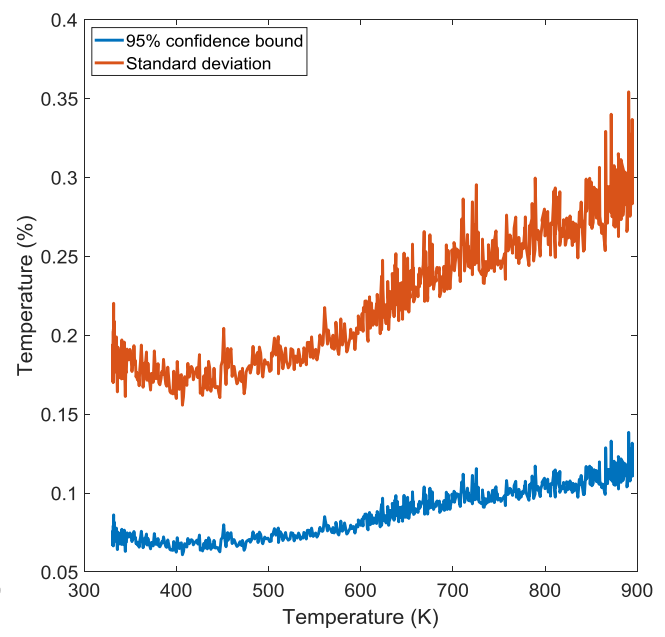

(b)

Figure 4: (a) A plot of the calibration curve for the powder surface emissivity with fit to the experimental data; (b) Quantification of the uncertainty in the temperature measurement vs temperature

\subsection{High-speed melt pool monitoring}

High-speed videos of the melt pool region were recorded at 50,000 frames per second for selected layers within each of the three sections of the tall cylinder. Images were acquired at $128 \times 128$ pixels, with a pixel size of $20 \mu \mathrm{m}$, and at two wavelengths, $700 \mathrm{~nm}$ and $950 \mathrm{~nm}$, to enable calculation of temperature using the coaxial two colour thermography methods described in [5]. The far higher frame rate and greater spatial resolution here dictate that only selected single layers can be recorded due to data rates and limited record times. The field of view exists just within the melt zone. One layer of the cylinder takes approximately $1 \mathrm{~s}$ to scan, producing 50,000 frames of footage per layer. When determining melt pool area and spatter characteristics, the first 33,500 frames of each layer recording were used in the analysis. During these frames the hatching of the cross-section takes place. For the remaining 16,500 frames in the layer, the machine is scanning the borders of the part and different laser parameters are used. Using the raw pixel intensity values, the melt pool is observed during processing and a qualitative assessment of the amount of spatter ejected during melting is made. Image processing and analysis was carried out on the intensity images using particle segmentation methods developed in [22]. The images were segmented using Otsu's method [23] combined with a Watershed Transform Function in order to distinguish spatter particles. This allowed for the labelling and counting of the number of ejected particles in each frame. In addition, a threshold criterion of $1400{ }^{\circ} \mathrm{C}$ was applied to the pixels of the calibrated temperature image, allowing the melt pool area to be calculated. These metrics were compared between the various recorded layers.

\subsection{Inter-layer cooling time}

As outlined in the introduction, the ILCT refers to the time interval between successive scans, layer-to-layer, of a given location on the powder bed. This is illustrated in 
Figure 5 with a plot of the thermal cycle undergone by a point on the component surface across three layers in a build. Here, the raw arbitrary pixel intensity measured by the camera is plotted and the signature associated with various process events occurring during these layers are annotated on the plot to demonstrate the ILCT concept.

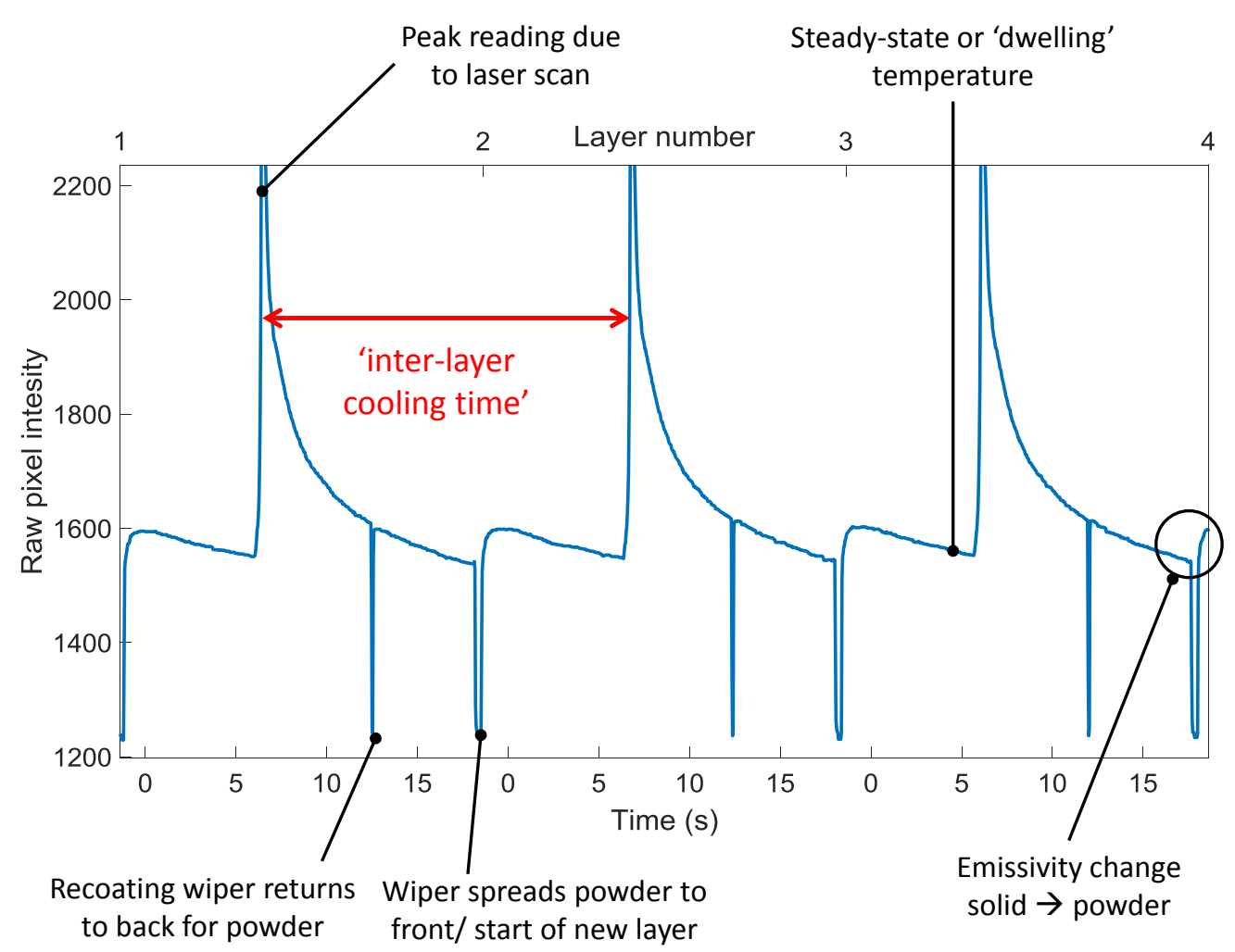

Figure 5: Annotated plot of the thermal cycle experienced at a location on the part surface during three layers of a build

The peak intensity reading occurs when the laser passes over the point of measurement. This is not representative of the peak melt pool temperature as the camera lacks the spatial and temporal resolution to resolve this. Instead, it is a spatially and temporally averaged peak value. The minimum pixel intensity readings are seen twice per layer. This is the powder spreading wiper blade passing over the measurement point, obscuring the view of the surface, and are not real drops in the surface temperature. The first minimum is caused by the wiper returning to collect fresh powder, then the second as it spreads powder across the new layer. Following the second minimum a slight increase in pixel intensity is observed. This is due to the newly spread powder surface having a higher emissivity than the consolidated layer below and therefore producing a higher IR emission intensity for a given temperature. The ILCT is annotated in red and shows the time span between two successive laser scans of the location on the part top surface. Finally, a dwelling temperature is also annotated which is the temperature of 
the point just before the laser falls incident upon it again.

\subsection{Build scenario to investigate ILCT}

To allow the ILCT to be varied in a controlled manner, the following build scenario was studied. Eight short cylinders of diameter $8 \mathrm{~mm}$ and height $20 \mathrm{~mm}$ were built alongside one tall cylinder of height $60 \mathrm{~mm}$. In the first $20 \mathrm{~mm}$ of the build, when all the cylinders are being scanned, the ILCT was approximately 20 seconds. In the middle section, when only the tall cylinder was being scanned, the ILCT was approximately 11 seconds. At $40 \mathrm{~mm}$ in height (or layer 800 out of 1200) eight short cylinders of the same dimensions were scanned at $5 \mathrm{~W}$ laser power to increase the scanning time and give an ILCT of approximately 20 seconds again. With the heavily reduced laser power no melting took place and only a very weak sintering effect occurred, meaning no supporting structures were needed for the upper small cylinders. An annotated photograph depicting the build is shown in Figure 6.

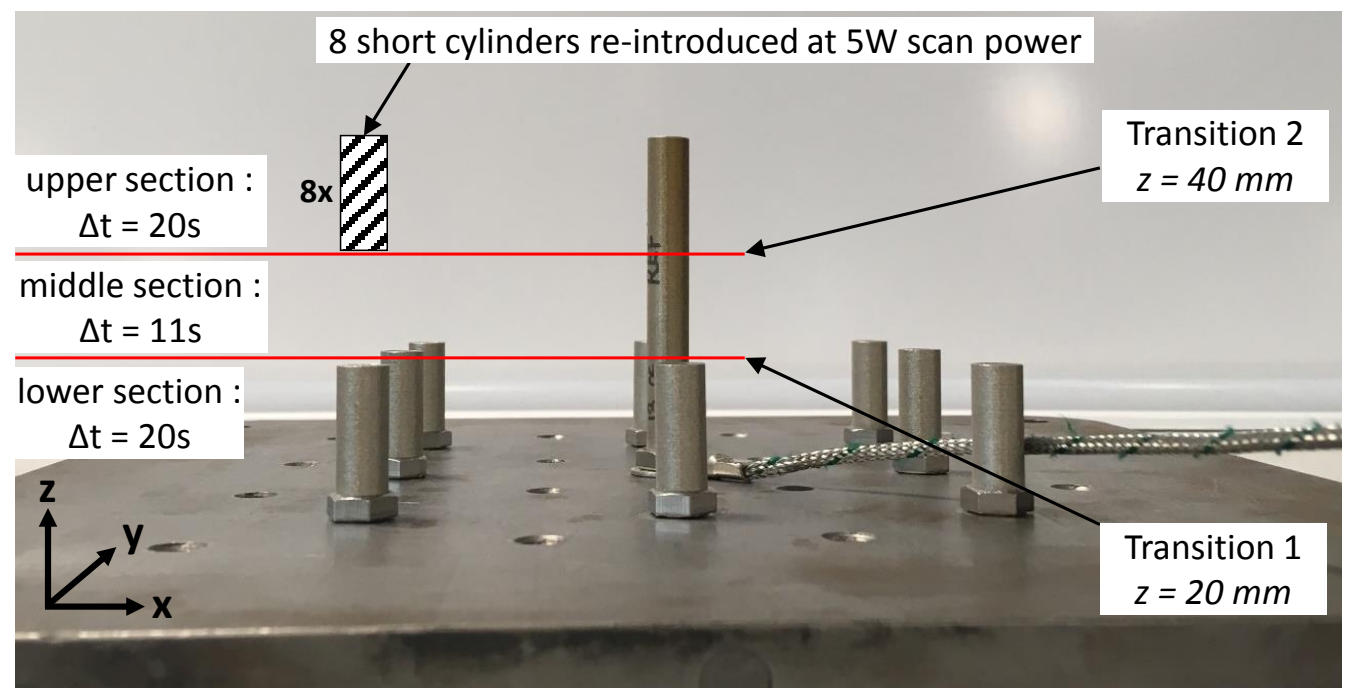

Figure 6: Annotated photograph depicting the build scenario

The transitions where the ILCT decreases then increases again are termed transition 1 and transition 2 respectively. Naming conventions describing the different build regions are also annotated on Figure 6 and these will be referred to throughout the paper. The cylinders were built with 316L stainless steel processed on a Renishaw AM250 machine. Default machine parameters were used except that a meander scan strategy [24] was used with no rotation between the layers to add clarity to microstructural observations. These are presented in Table 1. This allows the same position in the scanning process to be observed every layer when the part is sectioned.

As a control, a reference cylinder of identical specification to the tall cylinder was manufactured with no other components present in the build, using the same scanning parameters. This cylinder therefore had a constant ILCT of 11 seconds throughout the build, consistent with the middle section of the varied build. 
Table 1: The manufacturer default hatching parameters used to manufacture the cylinders. Note there was no rotation of the scanning direction between layers, contrary to the default setting of $67^{\circ}$.

\begin{tabular}{|c|c|c|}
\hline Parameter & Value & Unit \\
\hline \hline Laser power & 200 & $\mathrm{~W}$ \\
\hline Point distance & 60 & $\mu \mathrm{m}$ \\
\hline Exposure time & 80 & $\mu \mathrm{s}$ \\
\hline Hatch spacing & 110 & $\mu \mathrm{m}$ \\
\hline Spot size & 65 & $\mu \mathrm{m}$ \\
\hline Layer thickness & 50 & $\mu \mathrm{m}$ \\
\hline
\end{tabular}

\subsection{Microstructural and mechanical characterisation methods}

Following the build, the tall cylinder was sectioned in two along its axis and perpendicular to the scanning direction and then polished. Optical microscopy (OM) was then used to examine porosity and the microstructural changes associated with the ILCT. Several images of the polished surface were taken and then stitched together to give an overall image size of $20,200 \times 3120$ pixels, giving a resolution of approximately $3 \mu \mathrm{m}$ per pixel. Porosity in the cylinder was quantified using the find maxima function within FIJI[25], a version of the image processing software ImageJ. This calculates both the size and position of each pore on the sectioned surface by identifying local maxima (or minima). The pores were then divided into 12 bins by location in the cylinder height, with each bin being $5 \mathrm{~mm}$ tall. The relative density of each bin was then calculated.

To investigate grain size, morphology and orientation of the printed alloy in each section of the build, electron backscatter diffraction (EBSD) was carried out using a Zeiss Sigma 300 scanning electron microscope (SEM) operating at $20 \mathrm{kV}$. The step size used in generating the grain maps was $5.59 \mu \mathrm{m}$, giving a total scanned area of $2846 \times 2148 \mu \mathrm{m}^{2}$ which was large enough to be suitably representative of the microstructure in each of the three sections. Vickers hardness tests were also carried out at HV10 on a Zwick BTCFR2.5TN.D14 hardness tester to assess the impact on mechanical deformation behaviour. 40 locations were tested in each of the three sections with $0.5 \mathrm{~mm}$ spacing between points along the height, in a sawtooth formation between two different $\mathrm{x}$ co-ordinate locations $1 \mathrm{~mm}$ apart. The mean hardness value in each section was calculated, along with the standard deviation of the measurements. Standard error of the mean (SEM) statistical methodology was then used to evaluate $95 \%(2 \sigma)$ upper and lower confidence bounds in the hardness values for each section.

\section{Results and discussion}

\subsection{Surface temperature}

A typical IR image acquired during the build process is shown in Figure 7 . The image is taken part way through the processing of a layer in the lower section of the build. The laser has passed over 4 cylinders (three to the left and top middle) and is currently scanning the cylinder to the lower middle. Residual heat is visible in the 4 previously scanned cylinders and spatter is visible on the cylinder that is being scanned. Residual heat from the previous layer is also visible in the 4 remaining cylinders that are yet to be scanned. These cylinders will be approaching their dwell temperature. 


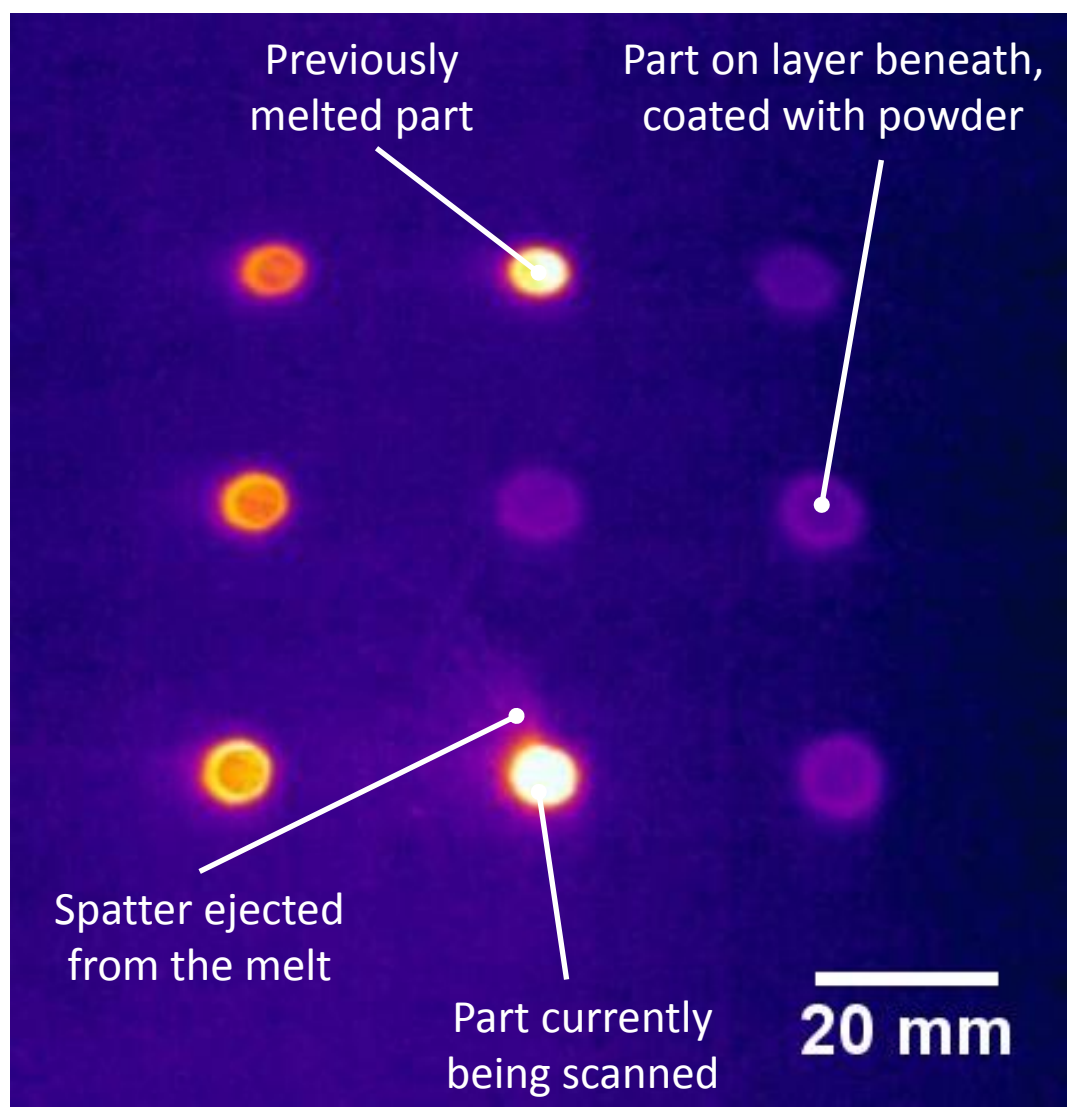

Figure 7: Example raw IR image acquired during scanning of the lower section

Measurements are taken from the central cylinder and temperatures are calculated using the previously derived calibration curves for both solid and powder. Figure 8 shows the temperature cycles for a number of layers with the long and short ILCT. Over 42 seconds, 2 layers are processed in the lower section and around 3.5 are processed in the middle section. This illustrates the difference in ILCT between the two sections. 

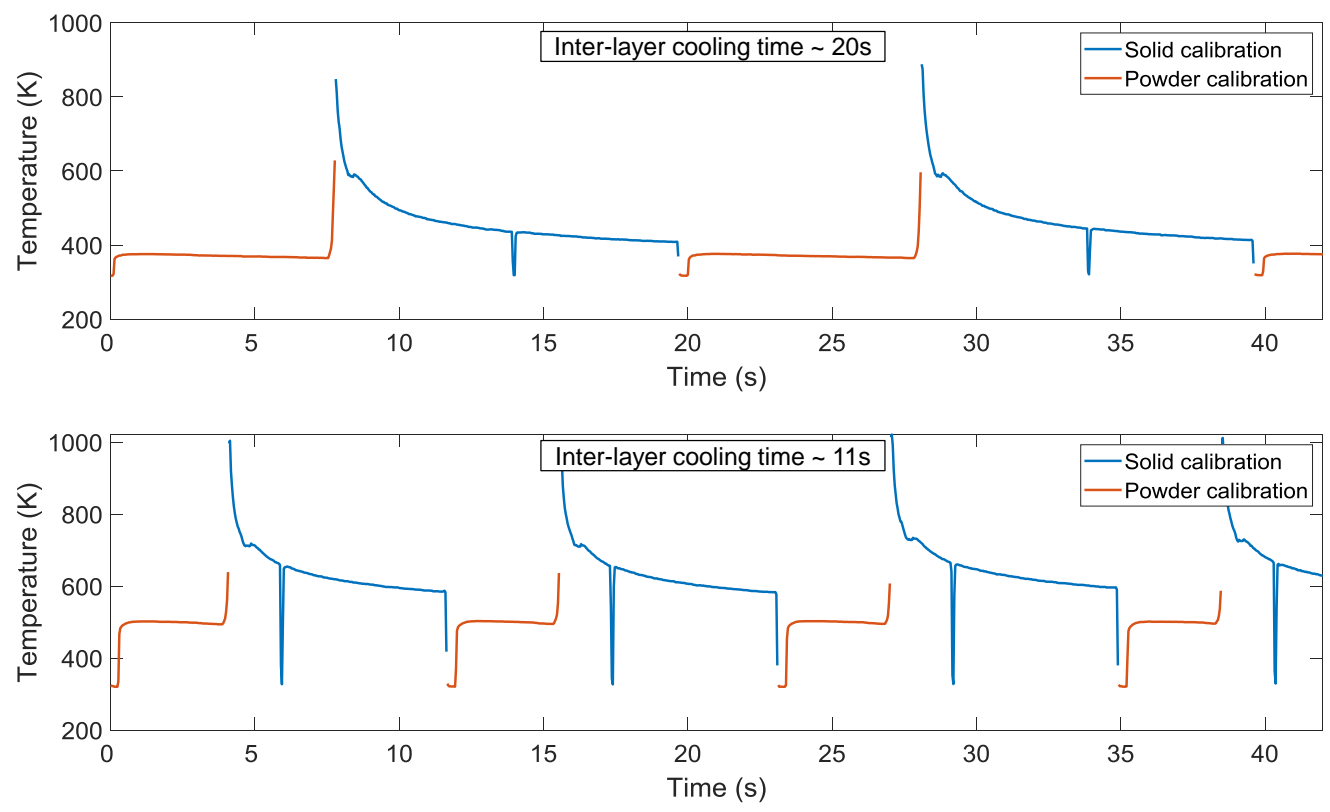

Figure 8: Calibrated surface temperature plot from the lower section (top) and middle section (bottom) recorded for 42 seconds by the IR camera, with both solid and powder calibration functions applied. The regions in the curve where the solid function is applied are plotted in blue and the regions where the powder function is applied in orange.

Once the temperature calibration function is applied, a temperature drop of around $40 \mathrm{~K}$ is seen when a new powder layer is spread, around $10 \%$ of the solid surface temperature. The powder is spread at approximately room temperature and will therefore extract heat from the solid surface when it is spread upon it. The heat required to raise the powder temperature will cause a drop in the overall surface temperature, explaining the observed drop. Note that this is the reverse trend to that which was seen in Figure 5 where the raw pixel intensity was plotted and the IR light emission actually increased after powder layer was spread. Due to the higher emissivity of the rougher powder surface, once the calibration functions are applied it is shown that the temperature actually decreases. This makes physical sense as the powder will be applied cold before being heated by the underlying part. The temperature is also seen to continue falling after the powder is spread due to the continued conduction of heat downwards via the baseplate [26]. A plot of the dwell surface temperature (the surface temperature just before the laser scans, see Figure 8) across the full build is shown in Figure 9. 


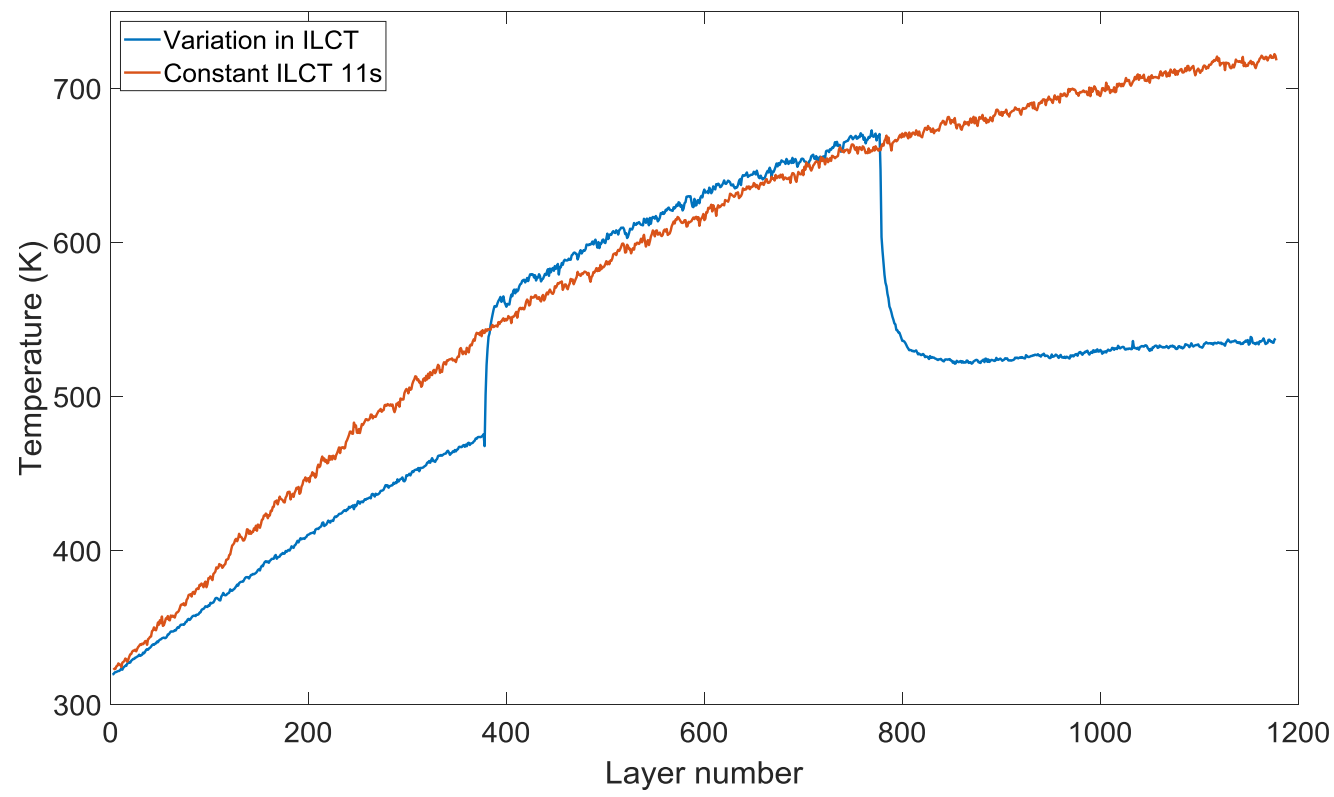

Figure 9: Surface temperature of the variation and reference samples taken just before the laser passes over each layer.

The increase in dwell temperature associated with the change in ILCT at layer 400 and 800 can clearly be seen. A sharp change in temperature of around $100 \mathrm{~K}$ is seen at layer 400 (transition 1) and a reduction of around $120 \mathrm{~K}$ at layer 800 (transition 2); these changes take place across 5 - 10 layers following the change in ILCT. After transition 2, the gradient of the surface temperature is flat, this indicates equilibrium has been reached and the rate of heat input is equal to the rate of heat loss via conduction through the baseplate and other losses to the surroundings. This thermal equilibrium may or may not reached in a build, depending upon changes in geometry and the resulting variations in ILCT. In this case, the reduction in ILCT during the middle region has increased the equilibrium temperature and the gradient shows the surface temperature increasing towards some higher value. It can also be seen that the temperature of both components is very low at the beginning of the build, due to the cold startup of the system. However, the surface temperature of the reference part increases at a much quicker rate and shows none of the signatures associated with the changes in ILCT. It should also be noted that although the Renishaw system uses a modulated (i.e. pulsed) continuous-wave laser, the pulsing is several orders of magnitude faster than the observed temperature trends. Therefore the effects observed here are equally applicable to unmodulated systems.

\subsection{Melt pool behaviour}

In addition to variations in surface temperature, changes in the melt pool behaviour were also observed as a result of the change in ILCT. These were captured and analysed using the high-speed monitoring setup detailed in Section 2.2. Still images of the melt pool taken from high speed camera recordings on layers 397 and 607 (i.e. either side of transition 1) are shown in a sequence of frames in Figure 10. The images are captured at 
the same $x y$ location on the part surface in each instance, over a time range of $488-494 \mathrm{~ms}$ through the scanning of the cylinder (the total time to scan the cylinder is approximately 1 second). 


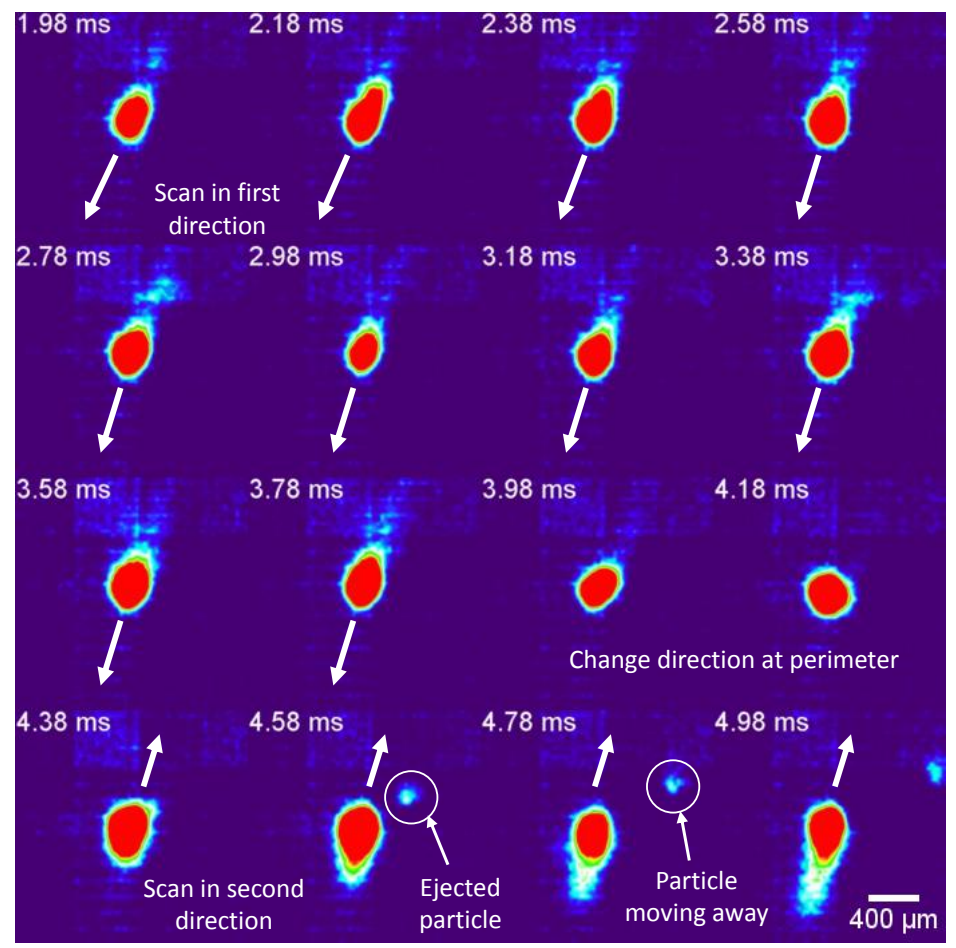

(a) Lower section (layer 397)

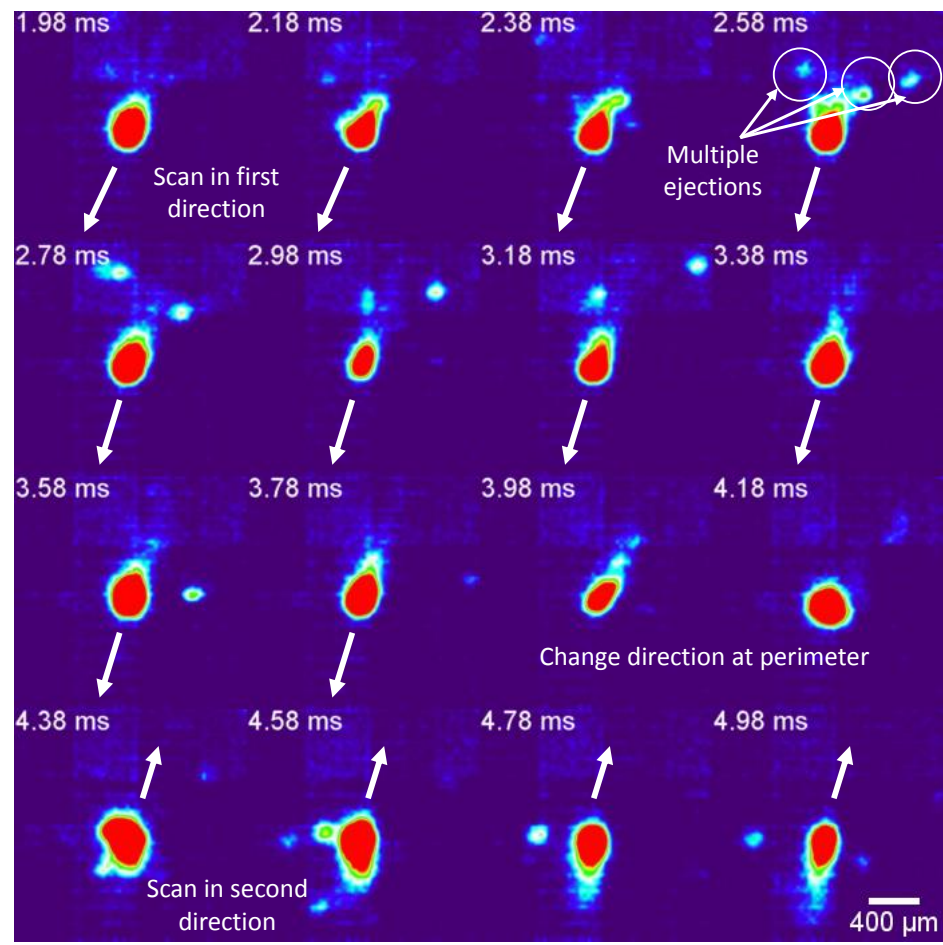

(b) Middle sectt5on (layer 607)

Figure 10: High speed videos of the melt pool, taken at the same time points and $x y$ location on the part surface, taken from layers 397 (a) and 607 (b) 
During this time period in scanning a layer of the cylinder, the melt pool approaches the perimeter of the circular cross-section, turns around and then begins scanning the adjacent track. This is consistent with expectation given the meander hatching strategy used. The action of the melt pool in turning around can clearly been seen in the still images as the comet tail of the melt pool reverses in direction following the frame at $4.38 \mathrm{~ms}$. Throughout all the sampled layers, the high speed videos of the melt pool revealed significantly more spatter in the middle section of the part compared with the lower section. During the 33,500 frames (or $0.67 \mathrm{~s}$ ) of footage of the hatching scan on layer $607,139,319$ hot ejected particles were detected, as opposed to 63,893 on layer 397 . Note that is not a count of the unique spatter particles ejected from the melt pool but is the total number of particles detected in all of the frames analysed. This provides a qualitative metric for comparison of the amount of spatter emitted in different layers. A plot of the total number of detected particles during the footage of the hatching on those layers analysed is shown in Figure 11.

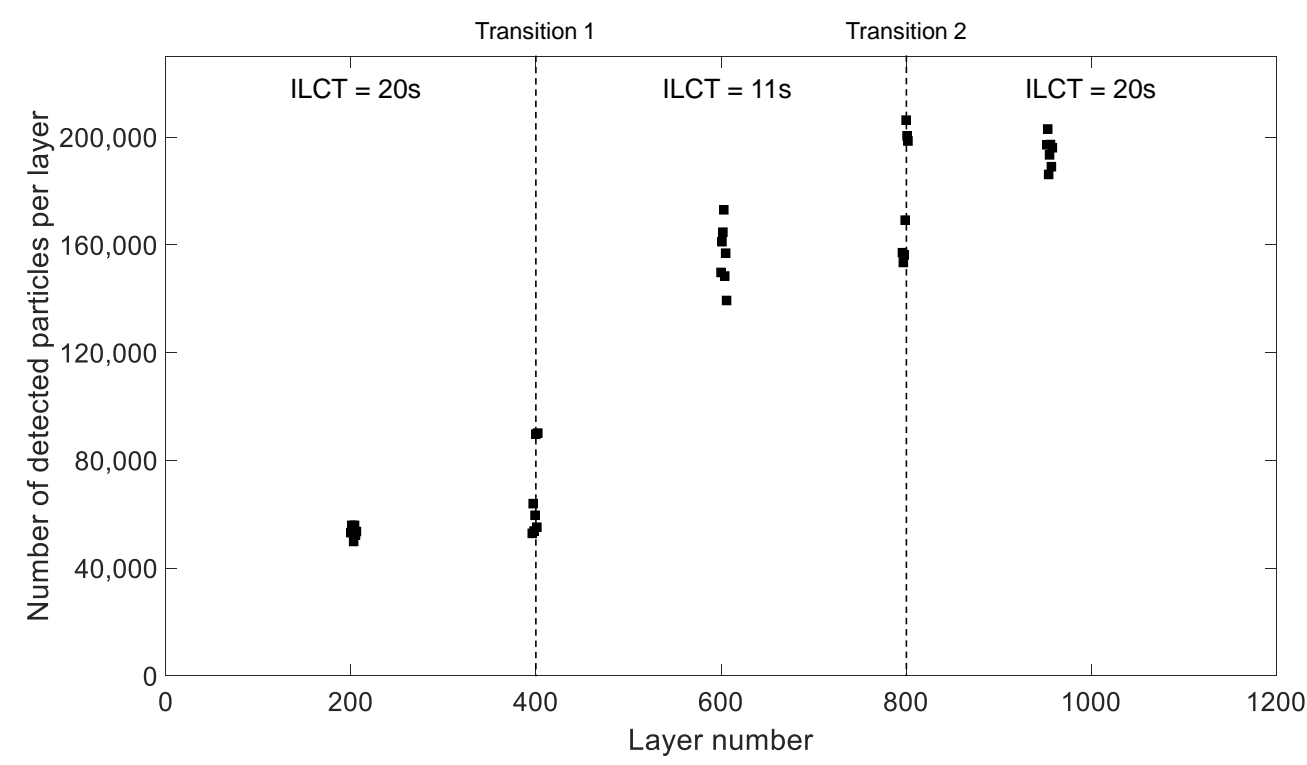

Figure 11: A plot of the total number of particles detected during the hatching scan (the first 33,500 of 50,000 frames) on the selected layers recorded by the high-speed camera system

As shown in Figure 9, the surface temperature of the part is increased in the middle section which seems to cause an increase in the number of spatter particles ejected. The trend does not follow the surface temperature plot in Figure 9 perfectly, however, and the spatter emission increases further beyond $40 \mathrm{~mm}$ in the build height once the ILCT increases again, despite the surface temperature falling. One possible reason for this is due to a change in the thickness of the delivered powder layer, caused by changes in the vertical thermal expansion and contraction of the part due to the differences in temperature. Spatter formation is a complex phenomenon which is not well understood and a simple relation with the surface temperature does not exist, although the weak correlation seen here suggests it is an influencing factor. 
In addition to the level of spatter, differences were seen in the melt pool size on the high-speed recordings of the layer hatching. A consistently larger melt pool area is measured across the entire 50,000 frame recording on layer 607 (in the middle section with the shorter ILCT) compared with layer 397 (in the lower section with the longer ILCT). The mean melt pool area for layer 607 was $0.0216 \mathrm{~mm}^{2}$, whereas on layer 397 it was $0.0194 \mathrm{~mm}^{2}$. Although the same amount of energy is delivered by the laser each layer, the temperature of the surface upon which it falls incident has been shown to vary in Figure 9. A larger melt pool area was measured on those layers recorded in the middle section with the higher surface temperature. As the laser is scanning material which is $100-150 \mathrm{~K}$ hotter, it is likely that more of the material will reach the melt threshold. Good agreement in the mean values between layers in the same region (and similar surface temperature) show that values are consistent and are not being significantly influenced by natural variations in powder spreading height. The mean melt pool area measured during hatching on selected layers is presented in Table 2, demonstrating the repeatability between measurements on adjacent layers. The size was determined in each frame and then a mean was calculated across the 33,500 frames of hatching. Finally, high frequency noise is present in the data and is attributed to the growing and shrinking of the melt pool as the laser pulses on and off.

Table 2: Mean melt pool area recorded during the 33500 frames of hatching scan on selected layers

\begin{tabular}{|c|c|c|}
\hline Layer number & Melt pool area $\left(\mathrm{mm}^{2}\right)$ & $95 \%$ confidence interval \\
\hline \hline 200 & 0.0206 & $\pm 3.9 \times 10^{-5}$ \\
\hline 201 & 0.0206 & $\pm 3.8 \times 10^{-5}$ \\
\hline 600 & 0.0243 & $\pm 5.0 \times 10^{-5}$ \\
\hline 607 & 0.0237 & $\pm 4.7 \times 10^{-5}$ \\
\hline 800 & 0.0248 & $\pm 5.2 \times 10^{-5}$ \\
\hline 803 & 0.0258 & $\pm 5.3 \times 10^{-5}$ \\
\hline
\end{tabular}

\subsection{Porosity and microstructure}

An image of the porosity from the sectioned cylinder with varying ILCT is shown in Figure 12 along with a histogram of the relative density of the 12 bins. The dwell surface temperature of each layer as plotted in Figure 9 is also overlaid. The equivalent taken for the reference component with constant ILCT is shown in Figure 13. 


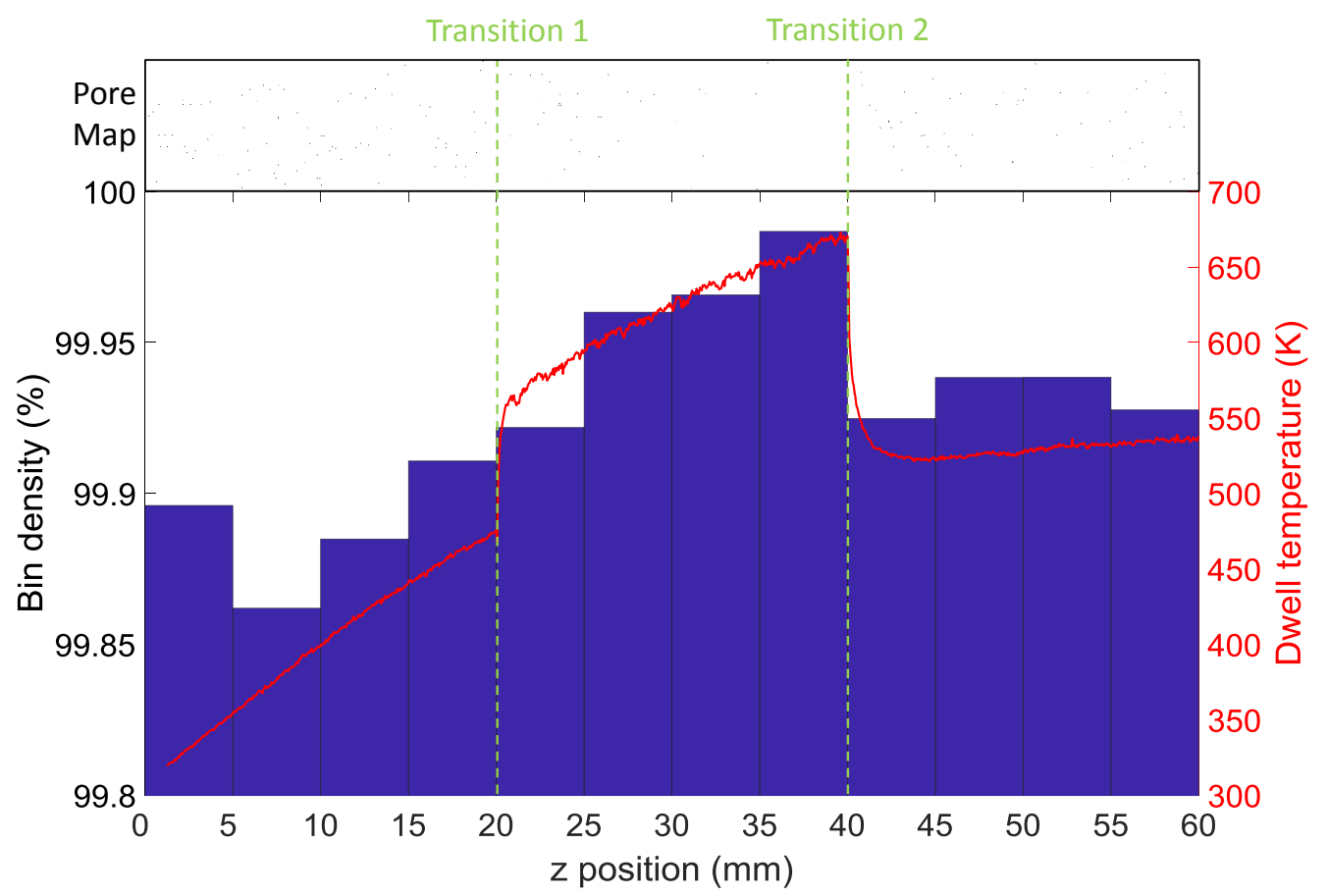

Figure 12: Porosity map taken at the centre of the sample with varied ILCT, below is the density histogram measured in $5 \mathrm{~mm}$ bins through the height of the sample. 


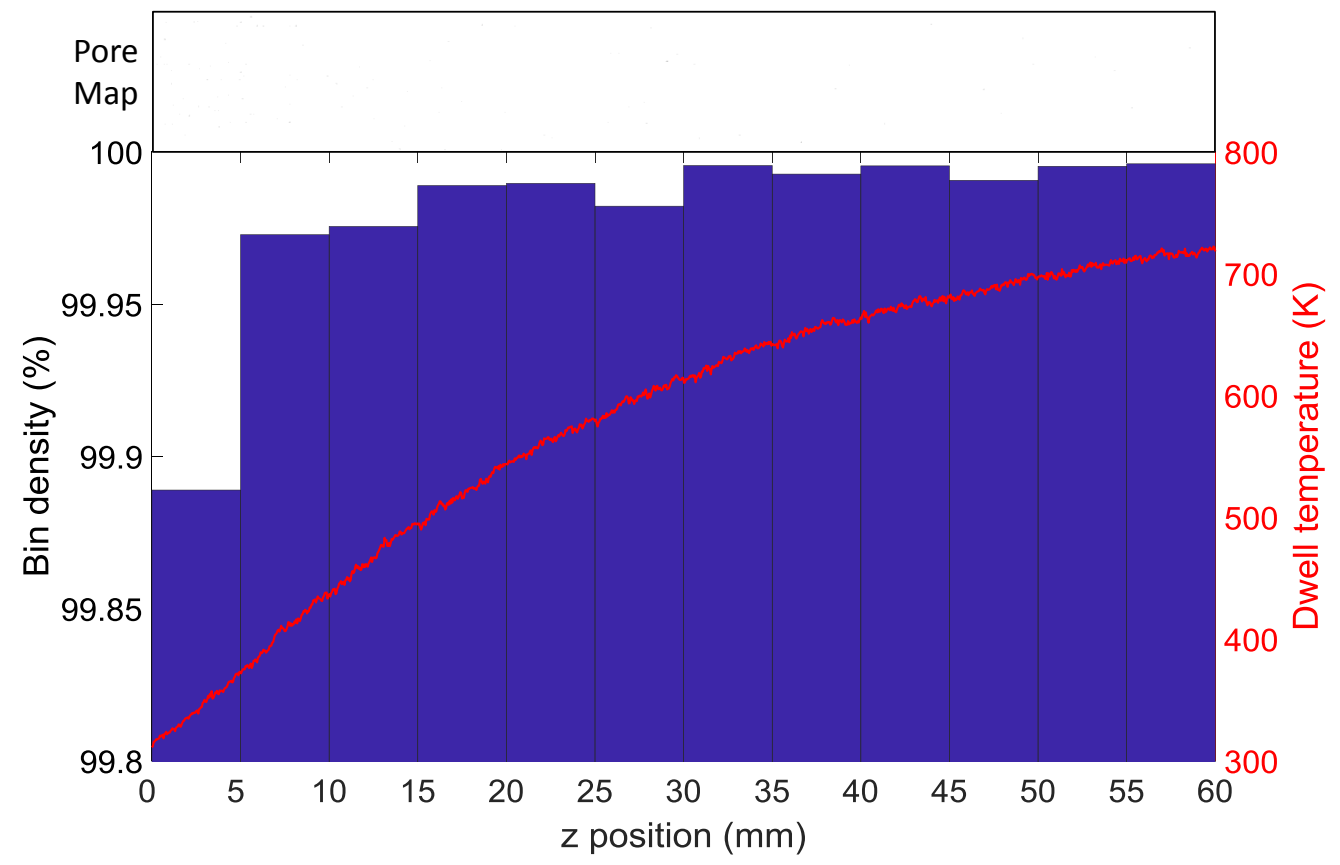

Figure 13: Porosity map taken at the centre of the reference sample, below is the relative density histogram measured in $5 \mathrm{~mm}$ bins through the height of the sample

Upon observing the trends in the surface temperature and relative density it can be seen that the two correlate, in both the variation and constant ILCT cases. When the surface temperature is lower, the porosity is generally greater. This would indicate that the pores are of lack of fusion mode [27] due to insufficient melting. To investigate this further, microscope images of the etched microstructure with a $5 \times$ objective lens are presented in the lower, middle and upper section of the varied ILCT part in Figure 14. Additionally, images from each region are also shown with a $20 \times$ objective lens in Figure 15 , to enable the melt pool morphology and porosity to be clearly seen. The percentage porosity of each section of the cylinder is also annotated on the images. 


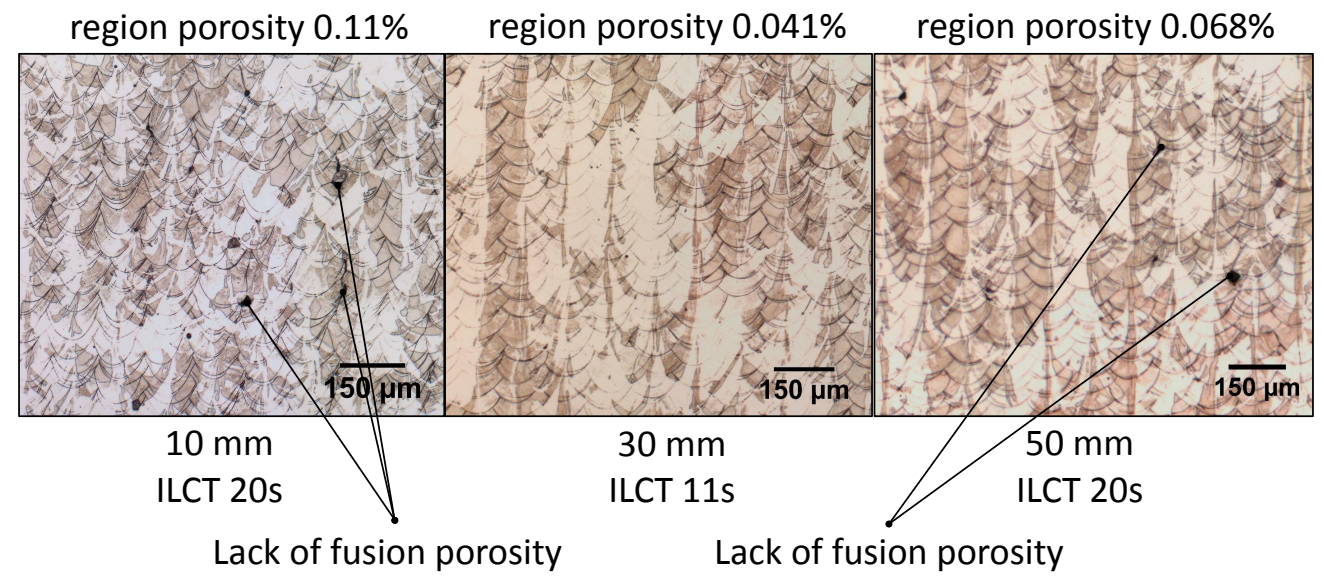

Figure 14: Etched OM images of the microstructure taken at the centre height of each of the three regions; lower $(10 \mathrm{~mm})$, middle $(30 \mathrm{~mm})$ and upper $(50 \mathrm{~mm})$. The percentage porosity of each region is annotated above (not a measure of porosity in the image, but the region as a whole)

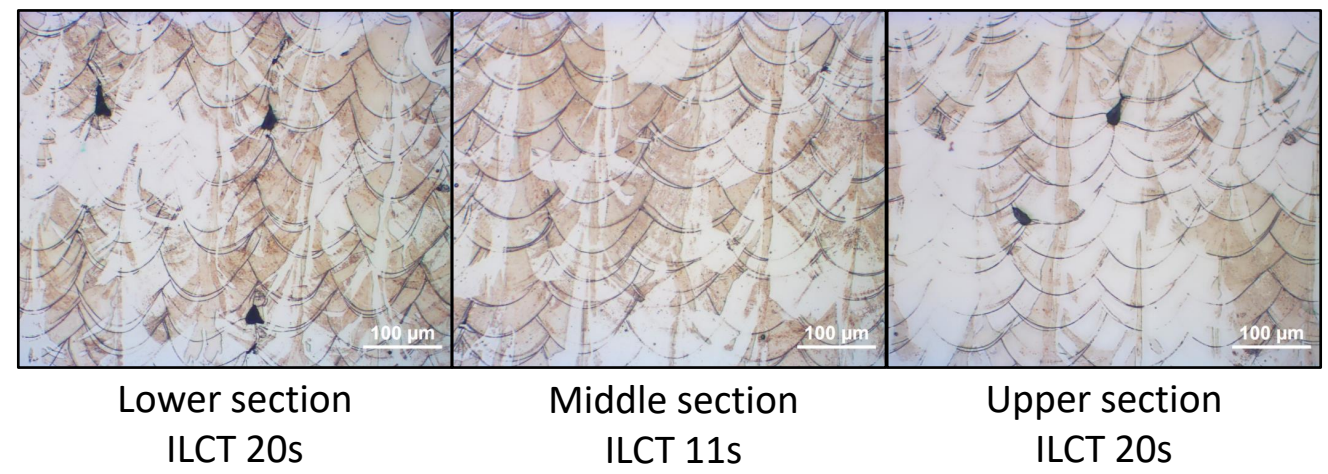

Figure 15: Higher magnification etched OM images of the microstructure taken from each of the three regions; lower, middle and upper. Lack of fusion porosity is visible in the lower and upper sections.

In the lower section, the melt pool morphology is seen to be narrow and elongated with clear lack of fusion between the tracks occurring in places, confirming this as the primary mechanism driving the higher porosity in this section. In the middle section the tracks appear rounder and overlapping at a lower angle. They are also stacked on top of one another more uniformly. The greater overlap has led to good consolidation and remedied the lack of fusion porosity. In the upper section, the melt pool morphology looks similar to the middle section however it can be seen that some lack of fusion between tracks has again occurred. This is consistent with the surface temperature measurements showing that the temperature had fallen again as the ILCT increased in the upper section. As the same amount of energy has fallen incident upon the surface but the surface is now cooler, the melt pool formed will be smaller and hence overlap less. This drives the lack of fusion porosity forming. This is backed up by the melt pool size measurements presented in 
Table 2, where a smaller melt pool was found on layer 397 , which is located within the bottom section where higher porosity was observed. While the pores found in between the tracks appear to be fairly small for typical lack of fusion defects, the direction of scanning should be noted. Lack of fusion pores tend to elongate in morphology along the scanning direction, between the tracks $[16,28,1]$. As the scanning direction does not rotate, the above micrographs are always showing a cross-section view of the pores, hence their long axis is not visible. The morphology of the above lack of fusion pores is consistent with those found in literature where the section view is roughly perpendicular to the scanning direction $[29,27]$. For this reason the trends in density shown in the histogram may in fact be greater than observed here but this is a limitation of $2 \mathrm{D}$ porosity area analysis.

The histogram plots of relative density illustrate the region of higher density in the middle section of the variation ILCT component where the temperature increased. In the upper section the relative density in each bin is lower as the porosity returned. On the other hand, the reference part shows very high density in all of the bins except the first. It is only for these first 200 layers that the surface temperature was very low due the cold start of the build, after which the surface temperature rose more rapidly due to the shorter ILCT. Some of the bins do not follow the hypothesis perfectly and this in part could be attributed to the fact the porosity measurements are only taken on a $2 \mathrm{D}$ plane and the formation of such pores can occur in any location through the thickness. Different bin widths were used in the plot and the same trend in porosity was always observed. The $5 \mathrm{~mm}$ bin width was chosen as an optimum balance between bins too large obscuring the transition in ILCT and bins too small containing insufficient number of pores. The density throughout both components as a whole is of a good standard for LPBF components, indicating that the process parameters are close to optimal.

As seen from the melt pool videos, the spatter is greater in the middle region when the surface is hotter. This is also the section where the part is found to have the highest relative density. Ejected spatter particles falling back to the part surface are frequently cited as a source of porosity in LPBF, characterised as a mechanism for lack of fusion porosity [30]. Here, an inverse correlation is seen, where higher levels of spatter yield higher density, as opposed to that in [30]. This implies that spatter is not the main cause of the porosity observed here. Spatter induced lack of fusion porosity is likely to be less prevalent in these cylinders due to their relatively small diameter, meaning much of the spatter generated lands outside of the part's surface. The spatter level is an indication of conditions in the melt pool zone. Recoil pressure at the surface of melt pool causes vortices in the gas above the melt pool, drawing powder towards the melt pool [31]. Some of the powder particles enter the melt pool and melt whilst others pass under the laser and are accelerated away by the gas flow caused by recoil pressure. Low levels of spatter indicate that less powder is being drawn towards the melt pool. For higher levels of spatter more powder is being drawn towards the melt pool, causing more powder to be melted overall and decreasing the level of porosity observed.

In contrast to the images seen in Figure 14 and Figure 15, the melt pool morphology in the reference sample with constant ILCT are fairly similar throughout. Etched micrographs displaying these at the same positions in height as above are shown in Figure 16. The morphology and overlap angle of the tracks is similar throughout the height. The tracks appear slightly less wide at the $10 \mathrm{~mm}$ location and this is consistent with the lower surface temperature at this build height. 


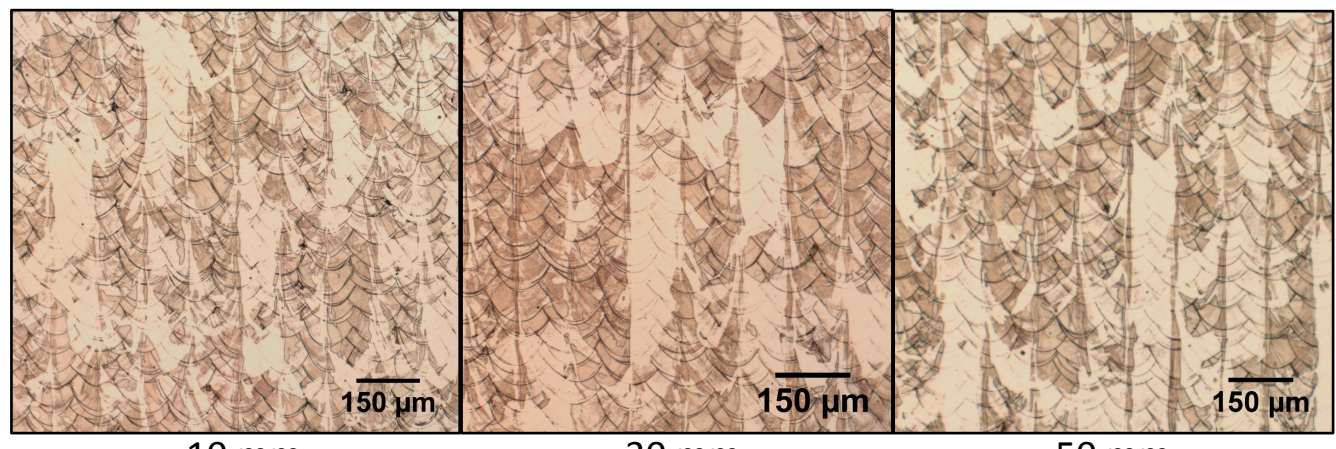

$10 \mathrm{~mm}$

$30 \mathrm{~mm}$

$50 \mathrm{~mm}$

Figure 16: Etched micrographs of the reference cylinder with constant ILCT at the same positions in the part as to be comparable with those in Figure 14

EBSD maps for each of the section of the build, along with grain size data extracted from each of the scans, are shown in Figure 17 . 

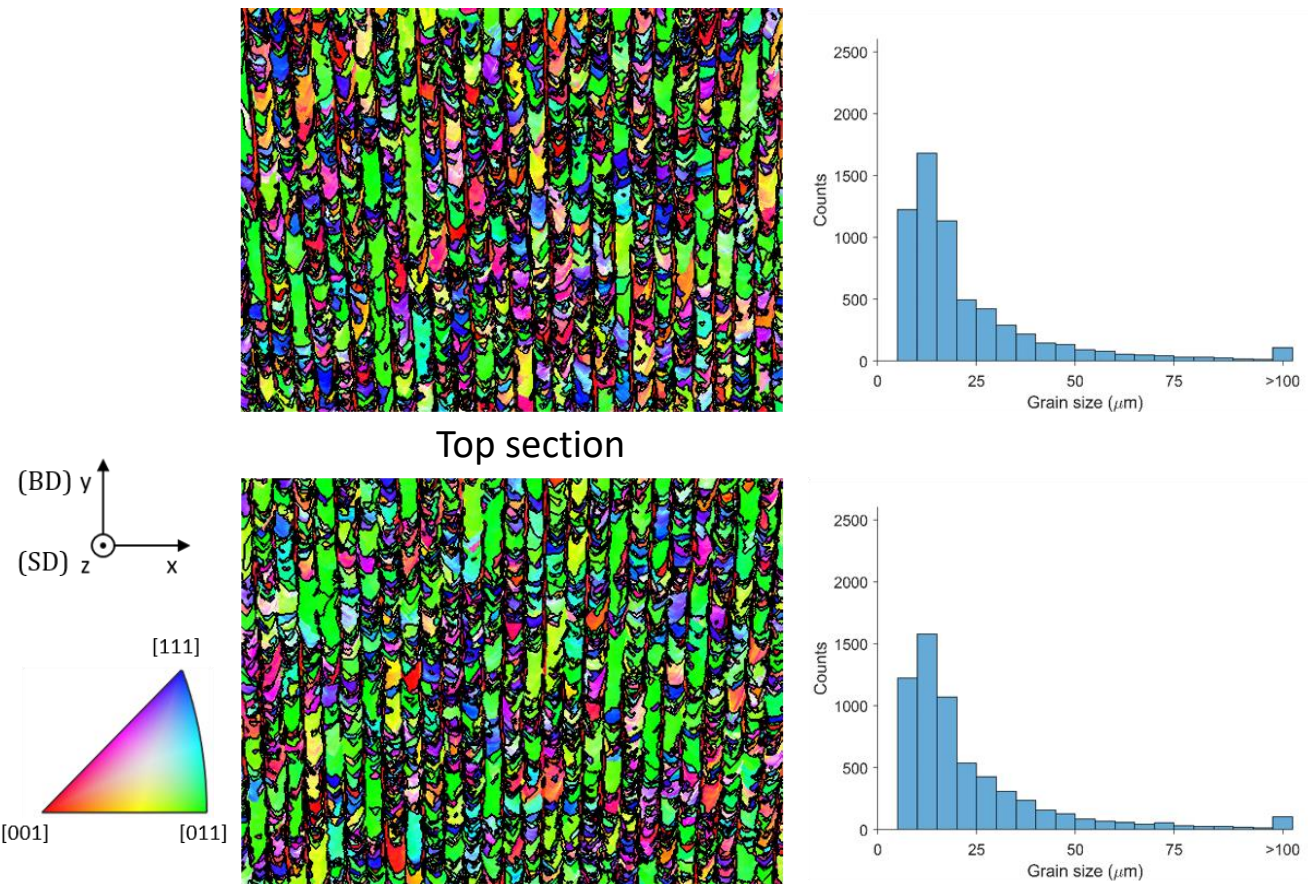

Middle section
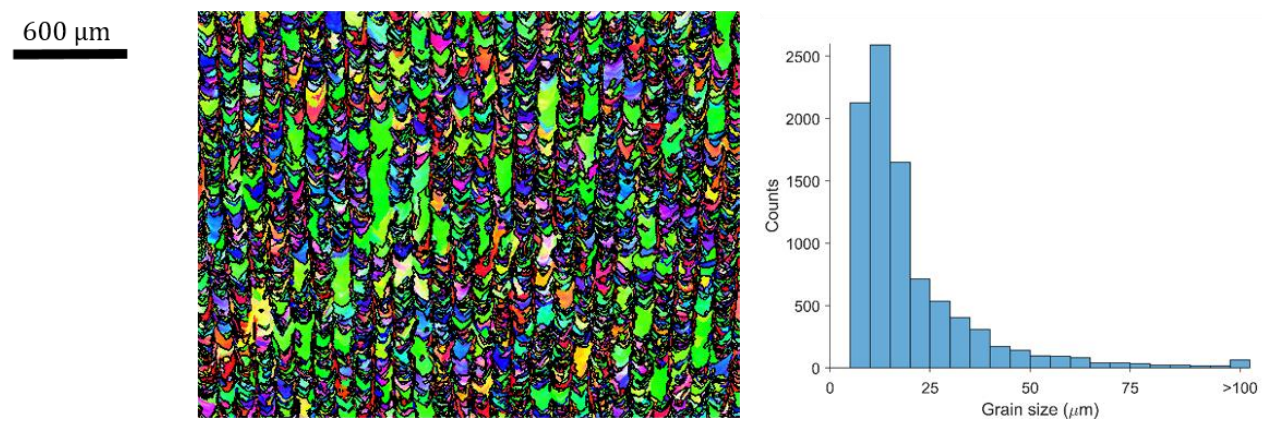

Bottom section

Figure 17: EBSD maps taken from the bottom, middle and top regions of the tall cylinder where inverse pole figure y (IPF-y) is parallel to the build direction (BD). Alongside the maps are histograms quantifying the grain size distribution.

The EBSD maps show qualitatively that the lower section is characterised by a high number of very fine grains, with very few coarse grains. In contrast, the middle section exhibits a significantly larger fraction of coarse grains. Finally, the grain structure of the upper section appears very similar to that of the middle section. The quantitative grain size data extracted from each of the scans (Figure 17, right column) confirm these trends. Furthermore, the average grain sizes were found to be of 20.4, 23.9 and $23.7 \mu \mathrm{m}$ for the lower, middle and upper sections, respectively.

The differences in grain size between the lower and middle sections correlate well 
with the ILCT differences. In the lower section, the long ILCT allows heat to disperse effectively from each newly-printed layer through the underlying build, lowering the temperature of the substrate. As the following layer is deposited, the temperature gradient $G$ between the new melt-pools and the substrate is high. In the middle section, on the other hand, there is less time for heat to disperse through the build, as the ILCT is reduced. The temperature of the substrate is higher and the temperature gradients within the new melt-pools are lower. In short, longer ILCTs result in higher temperature gradients. Since the scanning speed was not varied in the two sections, it can be assumed that the solidification rate $\mathrm{R}$ was equivalent in the lower and middle sections. Hence, differences in temperature gradients translate directly into different cooling rates (given by $G \times R$ ). It is well-known that high cooling rates inhibit grain coarsening and induce finer grains. This is why the lower section, printed with long ILCTs and thus high cooling rates, exhibits finer grains when compared to the middle section, characterised by shorter ILCTs and lower cooling rates.

Following the above observations, it might be expected that once the ILCT is increased back to the initial value in the upper section, the grain structure would resemble the one in the lower section. In fact, both the EBSD map and the quantitative grain size data show that the microstructure in the upper section resembles that of the middle section, with a relatively coarse grain size. This is partly due to the increase in temperature of the build as the print advances, which could balance the effect of the increase in ILCT. More importantly, the upper section effectively inherits the grain structure of the middle section via epitaxial growth. Indeed, epitaxial growth from existing grains is energetically favourable compared to the nucleation of new grains in solidifying melt-pools [32]. Hence, despite the longer ILCT, the coarse grain structure developed in the middle section tends to survive in the upper section thanks to epitaxial growth.

The solidification microstructure in the middle and upper sections, in terms of both grain morphology and crystallographic orientation, is consistent with that observed in a similar FCC alloy printed with the same scanning strategy in a previous work [32]. In particular, two kinds of long columnar grains that grew epitaxially through multiple layers along the build direction (BD) dominate the microstructure. Thin grains with one $<001>$ orientation parallel to the BD are found along the centreline of each melt pool column, while wider grains with one $\langle 110\rangle$ orientation parallel to the BD are found at the sides of each melt pool column. This particular microstructure is a direct consequence of the scanning strategy selected, and it is explained in greater detail in [32].

\subsection{Mechanical behaviour}

The results of hardness testing with respect to vertical position along the part are plotted in Figure 18. A weak correlation can be seen despite the large variation in values, showing a higher hardness in the lower region that decreases slightly with build height. 


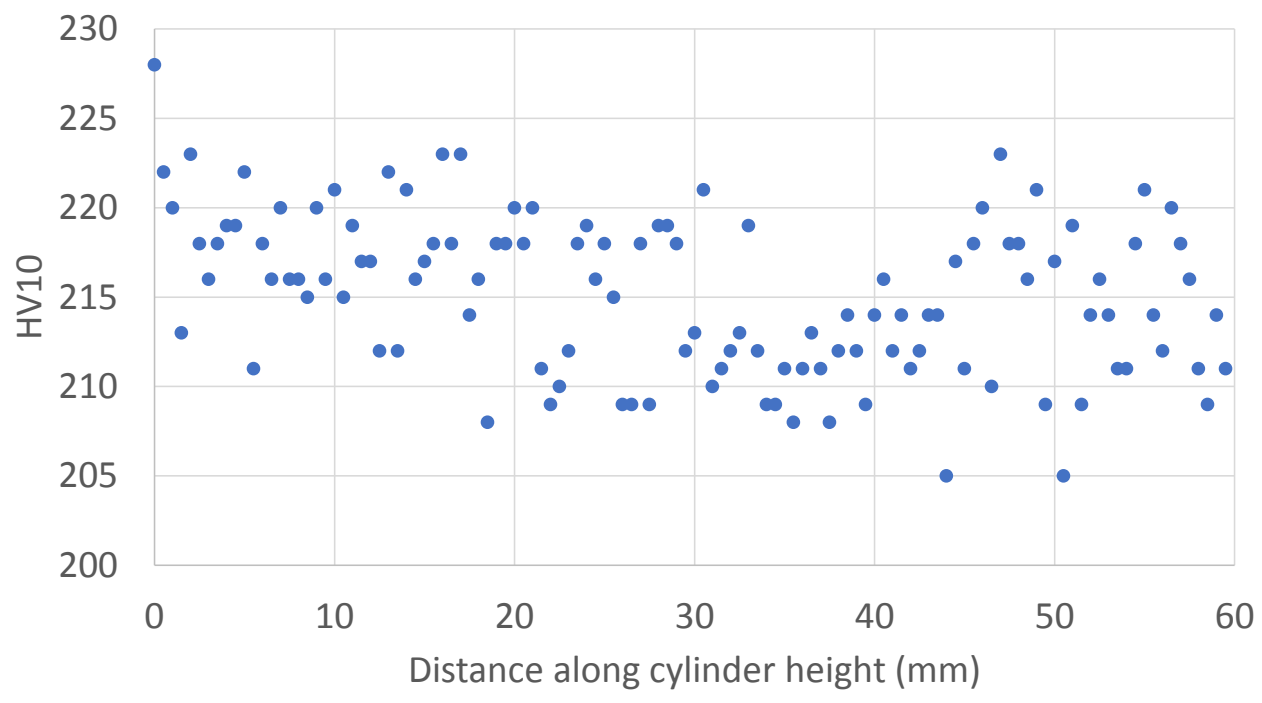

Figure 18: Plot of the hardness (HV10) value along the height of the sample $(z)$

The mean hardness values for the three regions are summarised in Table 3 . The mean hardness of the lower region is greater than in the middle region by $5 \mathrm{HV} 10$. The mean value of the upper section is also greater than middle section by $1 \mathrm{HV} 10$. Although these differences are small, the values suggest longer ILCT results in higher hardness values. Also shown in Table 3 are $95 \%$ confidence intervals. These represent the upper and lower bounds for the true mean value with $95 \%$ confidence (based on 40 samples). These intervals indicate that the increase in hardness observed between the lower and middle section is likely to be real, with a difference in true mean values. However, differences in hardness between the middle and upper sections may not be real and the sections may have the same true mean value.

These results are consistent with the microstructural information presented in Figure 17, which show a slightly smaller grain size in the lower section. Following a Hall-Petch type relation, a slightly higher hardness value would be expected in this region. Similarly, the difference in grain size between the middle and upper sections is very subtle as so no difference is observed in the hardness. Hardness testing is also highly localised and in compression only and so does not capture all aspects of the mechanical behaviour of a microstructure. Further differences in mechanical response between the three regions may be observed if the component was subject to different loading modes.

Table 3: Mean hardness values and 95\% upper and lower confidence bounds for values measured in each of the three sections

\begin{tabular}{cccc}
\hline Section & $\begin{array}{c}\text { Mean hardness } \\
\text { (HV10) }\end{array}$ & $\begin{array}{c}95 \% \text { upper } \\
\text { confidence bound }\end{array}$ & $\begin{array}{c}95 \% \text { lower } \\
\text { confidence bound }\end{array}$ \\
\hline Lower & 217.8 & 219.0 & 216.7 \\
\hline Middle & 213.3 & 214.5 & 212.1 \\
\hline Upper & 214.3 & 215.7 & 213.0 \\
\hline
\end{tabular}




\subsection{Factors affecting ILCT}

The effects on porosity, microstructure and mechanical properties detailed in this paper were found with a comparably subtle difference in ILCT of $9 \mathrm{~s}$. There is a general drive towards processing larger components and improving productivity through nesting multiple components [33]. With larger scan areas for bigger, or a greater number of, components the variation in ILCT could be upwards of 5 minutes. This would be expected to magnify the effects found in this paper, as greater changes in the rate of heat input would occur. Build pauses (for tasks such as topping up powder, changing filters, reinerting the chamber, etc) should be avoided at all costs as the sudden change in ILCT will have knock on effects for part quality. Preheating the build platform will reduce the surface temperature variations to some degree, but they will still be present as there is no control of surface temperature, only the underlying build platform temperature. Improvements to machine design to increase productivity, such as multi-laser systems, will tend to reduce the ILCT due to faster scanning times. However, there are additional complexities as the average rate of heat input will increase and also change depending how many lasers are firing concurrently.

\subsection{Implications for process parameter development}

Process parameters sets are effectively constant throughout a build, even if specific sets are used for surfaces (contour scans, overhangs, etc). The parameter sets used are material specific and are usually optimised iteratively using a set of simple geometries. These geometries are usually small to enable rapid turn around so that many combinations of parameters can be built and characterised. How well these optimised parameters transfer to real component geometries is uncertain. They may simply be the most tolerant parameter, able to cope with most build scenarios and geometries, but not necessarily the optimum for any particular layer or location within a build. In order to build material of optimum density and achieve uniform microstructure throughout, process parameters must be informed by the surface temperature of the component, with the aim of achieving consistent melt pool characteristics throughout the build. These parameters would be dynamic, adapting to the thermal field of the part, either by thermal simulations performed prior to building or in-process temperature measurements.

\subsection{Implications for qualification}

The two main factors influencing surface layer temperature and resulting part quality in LPBF are the geometry of a part and the average rate of heat input. The geometry of the part beneath the powder bed determines how quickly heat is conducted away from the build surface and the layer scanning time determines the average rate of heat input at the build surface. These two factors are changed whenever the build scene is changed. If more parts are added to a single build, the scanning time increases and the surface temperature drops. If different parts are nested together on the same build, changes in one part's scanning area will affect the overall ILCT and therefore the quality of all other parts on the same build. It is therefore not possible to qualify a LPBF component purely on the component in isolation. The component must be qualified when it is built as part of a particular build scene. If any component on that build scene changes, or other components are added/taken away, then it must be assumed that there are changes to all components on that build and every component on that build must be re-qualified. 
This negates some of the most attractive features about AM processes, particularly the ability to quickly make design changes. It also impacts productivity as flexibility is lost and only certified build scenes can be produced, rather than printing exactly what is needed. This further strengthens the case for in-situ monitoring and parameters that dynamically adapt to the build conditions.

\section{Conclusions}

This paper has for the first time highlighted surface layer temperature as a mechanism that can significantly affect build quality in Laser Powder Bed Fusion (LPBF). The effects of varying inter-layer cooling time (ILCT) during a build in LPBF were examined with a new in-situ infra-red (IR) thermography system to measure layer surface temperature during the build process. The measured surface temperature was shown to influence porosity, microstructure and mechanical properties of LPBF components. The work represents the first study of the impact of a change in ILCT during the build and also provides the first calibrated temperature measurements of the problem. The following conclusions are drawn:

- In very common build scenarios, such as changing the number of parts processed in a LPBF build, the ILCT and surface temperature varies throughout the build due to changes in scanning time and part geometry

- Reduction in the ILCT causes the surface temperature of the component to rise, and vice versa.

- Spatter formation is increased when surface temperatures are higher and appears to be inversely related to porosity.

- Different ILCTs lead to different melt pool shapes, of which non-optimal shapes were identified as a cause of lack of fusion porosity. Shorter ILCT led to optimal melt pool shapes and eliminated such porosity.

- Greater grain growth and preferential orientation were observed as a result of shorter ILCT, and therefore higher surface temperatures resulting in a lower thermal gradient $G$. A small effect on hardness was seen and attributed to the reduced grain size in the short ILCT sections.

The implication of these conclusions are that qualification of a component must also be done on a build basis, not for a single component in isolation. Further, to build material of optimum density and achieve a uniform microstructure throughout, the surface temperature of a component must be used to inform process parameters. This understanding allows for an increased confidence in the consistency and structural integrity of LPBF components.

\section{Acknowledgements}

We thank AWE plc (contract 30338995) for funding Richard Williams and Tobias Rønneberg for their PhD studies and Paul Hooper's research fellowship. 


\section{References}

[1] T. Debroy, H. L. Wei, J. S. Zuback, T. Mukherjee, J. W. Elmer, J. O. Milewski, A. M. Beese, A. Wilson-Heid, A. De, W. Zhang, Additive manufacturing of metallic components - Process, structure and properties, Progress in Materials Science (92) (2018) 112-224 (mar 2018). doi:10.1016/j.pmatsci.2017.10.001.

[2] W. E. King, A. T. Anderson, R. M. Ferencz, N. E. Hodge, C. Kamath, S. A. Khairallah, A. M. Rubenchik, Laser powder bed fusion additive manufacturing of metals; physics, computational, and materials challenges, Applied Physics Reviews 2 (4) (2015) 041304 (dec 2015). doi:10.1063/1.4937809.

[3] S. K. Everton, M. Hirsch, P. Stravroulakis, R. K. Leach, A. T. Clare, Review of in-situ process monitoring and in-situ metrology for metal additive manufacturing, Materials \& Design 95 (2016) 431-445 (2016). doi:10.1016/j.matdes.2016.01.099.

[4] M. Grasso, B. M. Colosimo, Process defects and monitoring methods in metal powder bed fusion: a review, Measurement Science and Technology 28 (4) (2017) 44-69 (apr 2017). doi:10.1088/1361$6501 / \mathrm{aa} 5 \mathrm{c} 4 \mathrm{f}$.

[5] P. A. Hooper, Melt pool temperature and cooling rates in laser powder bed fusion, Additive Manufacturing 22 (2018) 548-559 (aug 2018). doi:10.1016/J.ADDMA.2018.05.032.

[6] L. Parry, I. A. Ashcroft, R. D. Wildman, Understanding the effect of laser scan strategy on residual stress in selective laser melting through thermo-mechanical simulation, Additive Manufacturing 12 (2016) 1-15 (2016). doi:10.1016/j.addma.2016.05.014.

[7] W. E. King, H. D. Barth, V. M. Castillo, G. F. Gallegos, J. W. Gibbs, D. E. Hahn, C. Kamath, A. M. Rubenchik, Observation of keyhole-mode laser melting in laser powder-bed fusion additive manufacturing, Journal of Materials Processing Technology 214 (12) (2014) 2915-2925 (dec 2014). doi:10.1016/J.JMATPROTEC.2014.06.005.

[8] D. B. Hann, J. Iammi, J. Folkes, A simple methodology for predicting laser-weld properties from material and laser parameters, Journal of Physics D: Applied Physics 44 (44) (2011) 445401 (oct 2011). doi:10.1088/0022-3727/44/44/445401.

[9] S. A. Khairallah, A. T. Anderson, A. Rubenchik, W. E. King, Laser powder-bed fusion additive manufacturing: Physics of complex melt flow and formation mechanisms of pores, spatter, and denudation zones, Acta Materialia 108 (2016) 36-45 (April 2016). doi:10.1016/j.actamat.2016.02.014.

[10] M. H. Farshidianfar, A. Khajepour, A. Gerlich, Real-time control of microstructure in laser additive manufacturing, The International Journal of Advanced Manufacturing Technology 82 (5-8) (2016) 1173-1186 (feb 2016). doi:10.1007/s00170-015-7423-5.

[11] M. Ma, Z. Wang, X. Zeng, A comparison on metallurgical behaviors of 316L stainless steel by selective laser melting and laser cladding deposition, Materials Science and Engineering: A 685 (2017) 265-273 (feb 2017). doi:10.1016/j.msea.2016.12.112.

[12] Y. M. Wang, T. Voisin, J. T. McKeown, J. Ye, N. P. Calta, Z. Li, Z. Zeng, Y. Zhang, W. Chen, T. T. Roehling, R. T. Ott, M. K. Santala, P. J. Depond, M. J. Matthews, A. V. Hamza, T. Zhu, Additively manufactured hierarchical stainless steels with high strength and ductility, Nature Materials 17 (1) (2017) 63-71 (oct 2017). doi:10.1038/nmat5021.

[13] A. Yadollahi, N. Shamsaei, S. M. Thompson, A. Elwany, L. Bian, Mechanical and Microstructural Properties of Selective Laser Melted 17-4 PH Stainless Steel, Volume 2A: Advanced Manufacturing (November) (2015) V02AT02A014 (2015). doi:10.1115/IMECE2015-52362.

[14] E. R. Denlinger, J. C. Heigel, P. Michaleris, T. Palmer, Effect of inter-layer dwell time on distortion and residual stress in additive manufacturing of titanium and nickel alloys, Journal of Materials Processing Technology 215 (2015) 123-131 (jan 2015). doi:10.1016/J.JMATPROTEC.2014.07.030.

[15] Effect of inter-layer cooling time on distortion and mechanical properties in metal additive manufacturing.

[16] A. Yadollahi, N. Shamsaei, Additive manufacturing of fatigue resistant materials: Challenges and opportunities, International Journal of Fatigue 98 (2017) 14-31 (may 2017). doi:10.1016/j.ijfatigue.2017.01.001

[17] E. W. Lui, W. Xu, A. Pateras, M. Qian, M. Brandt, New Development in Selective Laser Melting of Ti-6Al-4V: A Wider Processing Window for the Achievement of Fully Lamellar a + b Microstructures, JOM 69 (12) (2017) 2679-2683 (dec 2017). doi:10.1007/s11837-017-2599-9.

[18] W. Xu, E. W. Lui, A. Pateras, M. Qian, M. Brandt, In situ tailoring microstructure in additively manufactured Ti-6Al-4V for superior mechanical performance (2017). doi:10.1016/j.actamat.2016.12.027.

[19] J. L. Bartlett, F. M. Heim, Y. V. Murty, X. Li, In situ defect detection in selective laser melt- 
ing via full-field infrared thermography, Additive Manufacturing 24 (2018) 595-605 (dec 2018). doi:10.1016/J.ADDMA.2018.10.045.

[20] N. Boone, C. Zhu, C. Smith, I. Todd, J. Willmott, Thermal near infrared monitoring system for electron beam melting with emissivity tracking, Additive Manufacturing 22 (2018) 601-605 (aug 2018). doi:10.1016/J.ADDMA.2018.06.004.

[21] H. Krauss, C. Eschey, M. F. Zaeh, Thermography for Monitoring the Selective Laser Melting Process, in: Proceedings of Solid Freeform Fabrication Symposium, 2012 (2012).

[22] C. Jones, D. B. Hann, K. T. Voisey, S. Aitken, Application of high speed filming techniques to the study of rearwards melt ejection in laser drilling, Tech. rep. (2016). doi:10.2351/1.4983269.

[23] N. Otsu, A Threshold Selection Method from Gray-Level Histograms, IEEE Transactions on Systems, Man, and Cybernetics 9 (1) (1979) 62-66 (jan 1979). doi:10.1109/TSMC.1979.4310076.

[24] Design for metal am - a beginner's guide, Tech. rep., Renishaw plc (2017).

[25] J. Schindelin, I. Arganda-Carreras, E. Frise, V. Kaynig, M. Longair, T. Pietzsch, S. Preibisch, C. Rueden, S. Saalfeld, B. Schmid, J.-Y. Tinevez, D. J. White, V. Hartenstein, K. Eliceiri, P. Tomancak, A. Cardona, Fiji: an open-source platform for biological-image analysis, Nature Methods 9 (7) (2012) 676-682 (jul 2012). doi:10.1038/nmeth.2019.

[26] R. J. Williams, C. M. Davies, P. A. Hooper, A pragmatic part scale model for residual stress and distortion prediction in powder bed fusion, Additive Manufacturing 22 (2018) 416-425 (aug 2018). doi:10.1016/J.ADDMA.2018.05.038.

[27] J. A. Cherry, H. M. Davies, S. Mehmood, N. P. Lavery, S. G. R. Brown, J. Sienz, Investigation into the effect of process parameters on microstructural and physical properties of 316L stainless steel parts by selective laser melting, The International Journal of Advanced Manufacturing Technology 76 (5-8) (2015) 869-879 (feb 2015). doi:10.1007/s00170-014-6297-2.

[28] B. Zhang, Y. Li, Q. Bai, Defect Formation Mechanisms in Selective Laser Melting: A Review, Chinese Journal of Mechanical Engineering (English Edition) 30 (3) (2017) 515-527 (May 2017). doi:10.1007/s10033-017-0121-5.

[29] M. Zhang, C.-N. Sun, X. Zhang, P. C. Goh, J. Wei, D. Hardacre, H. Li, Fatigue and fracture behaviour of laser powder bed fusion stainless steel 316L: Influence of processing parameters, Materials Science \& Engineering A 703 (2017) 251-261 (Aug 2017). doi:10.1016/j.msea.2017.07.071.

[30] Y. Liu, Y. Yang, S. Mai, D. Wang, C. Song, Investigation into spatter behavior during selective laser melting of AISI 316L stainless steel powder, Materials \& Design 87 (2015) 797-806 (dec 2015). doi:10.1016/j.matdes.2015.08.086.

[31] M. J. Matthews, G. Guss, S. A. Khairallah, A. M. Rubenchik, P. J. Depond, W. E. King, Denudation of metal powder layers in laser powder bed fusion processes, Acta Materialia 114 (2016) 33-42 (aug 2016). doi:10.1016/J.ACTAMAT.2016.05.017.

[32] A. Piglione, B. Dovgyy, C. Liu, C. Gourlay, P. Hooper, M. Pham, Printability and microstructure of the CoCrFeMnNi high-entropy alloy fabricated by laser powder bed fusion, Materials Letters 224 (2018) 22-25 (aug 2018). doi:10.1016/J.MATLET.2018.04.052.

[33] S. Davies, Betatype's volume additive manufacture of automotive headlight parts saves days in time and thousands of pounds, TCT magazine (aug 2018).

URL https://www.tctmagazine.com/3d-printing-news/betatype-volume-additivemanufacture-automotive-headlight-parts / 